\title{
THE TERM STRUCTURE OF INTEREST \\ RATES IN A PURE EXCHANGE ECONOMY WITH HETEROGENEOUS INVESTORS
}

\author{
Jiang Wang
}

Working Paper No. 5172

\author{
NATIONAL BUREAU OF ECONOMIC RESEARCH \\ 1050 Massachusetts Avenue \\ Cambridge, MA 02138 \\ July 1995
}

I thank Petr Adamek, Fischer Black, George Constantinides, John Heaton, Chi-fu Huang, Andrew Lo, John Long (the editor), Angel Serrat Tubert, seminar participants at Baruch College, Carnegie-Mellon University, Columbia University, Princeton University, University of California at Berkeley, University of Chicago, University of Wisconsin at Madison, and especially a referee for helpful comments. Support from the Laboratory of Financial Engineering, the Taiwan Program and the IFSRC at the Sloan School of Management is gratefully acknowledged. This paper is part of NBER's research program in Asset Pricing. Any opinions expressed are those of the author and not those of the National Bureau of Economic Research.

() 1995 by Jiang Wang. All rights reserved. Short sections of text, not to exceed two paragraphs, may be quoted without explicit permission provided that full credit, including (C) notice, is given to the source. 


\title{
THE TERM STRUCTURE OF INTEREST \\ RATES IN A PURE EXCHANGE ECONOMY WITH HETEROGENEOUS INVESTORS
}

\begin{abstract}
This paper presents an equilibrium model of the term structure of interest rates when investors have heterogeneous preferences. The basic model considers a pure exchange economy of two classes of investors with different (but constant) relative risk-aversion and gives closedform solutions to bond prices. We use the model to examine the effect of preference heterogeneity on the behavior of bond yields. Extensions to cases of more than two investors are also considered.
\end{abstract}

\section{Jiang Wang}

Sloan School of Management, E52-448

MIT

50 Memorial Drive

Cambridge, MA 02142-1347

and NBER 


\section{Introduction}

Existing models of equilibrium term structure of interest rates are often based on the representative agent framework with specific parametric assumptions about the preferences of the representative agent. For example, the well known model of Cox, Ingersoll and Ross (1985a) [CIR, thereafter] assumes that the representative agent has logarithmic preferences and faces a production opportunity with its expected return following a "square-root" process. ${ }^{1}$ When the financial market is complete [in the sense of Harrison and Kreps (1979)], a representative agent can be constructed whose marginal utility under the given process of aggregate consumption determines the equilibrium security prices [see, e.g., Constantinides (1982)]. However, the preference of the representative agent is in general quite complicated even when the preferences of individual investors are simple [see, e.g., Dumas (1989)]. ${ }^{2}$ It should be derived from the primitives of the economy such as the individual preferences as part of the equilibrium analysis, instead of being assumed. Thus, even though strong assumptions about the representative agent's preferences can lead to simple bond pricing formulas, they are often too restrictive to reflect the effect of any investor heterogeneity on the behavior of bond prices. Furthermore, starting from the representative agent without explicitly modeling the interaction among individual investors leaves out any implications on quantities [such as the amount investors borrow and lend] and how they are related to bond prices and interest rates. In addition, linking bond prices and dis-aggregated variables such as the amount of borrowing and the distribution of consumption and wealth among investors makes the model more appealing empirically. Note that the representative agent models only relate the bond prices to the underlying state variables which may not be directly observable. By explicitly modeling individual investors, we can relate the bond prices to the dis-aggregated variables which may be directly observable. Thus they can be used as instruments for the underlying state variables in any empirical implementation of the model.

In this paper, we consider a simple pure exchange economy with two classes of investors who have time-additive, state-independent and constant relative risk-aversion [CARA] preferences with risk-aversion coefficients $a_{1}$ and $a_{2}$, respectively, where $a_{1}>a_{2}$. Equilibrium bond prices

\footnotetext{
${ }^{1}$ See also Longstaff and Schwartz (1992) and Sun (1992).

${ }^{2}$ Rubinstein (1974) considers special cases of investor preferences when the representative agent's preference exhibits simple forms.
} 
and yields are solved in closed-form. The main goal of the paper is to examining how the heterogeneity in preferences may affect the behavior of the term structure of interest rates. In particular, the equilibrium term structure of interest rate with both classes of investors present is compared with the term structure with only one class of investors.

In general, the yield curve with both classes of investors behave differently from the two yield curves with respectively only one of the two classes of the investors present in the economy. Consider the simple case where the aggregate consumption follows a geometric Brownian motion, hence its growth will be i.i.d. over time. In the worlds populated by only one class of investors respectively, their consumption is simply the aggregate consumption. Since their utility function is isoelastic, the growth of their marginal utility will then be i.i.d. over time also. Thus the interest rates will be constant over time and the term structure will be flat, independent of the level of aggregate consumption, [see, e.g., Stapleton and Subrahmanyam (1990) and McCulloch (1993)]. When both classes of investors are present, however, the growth of investors' marginal utility will be endogenous and non-i.i.d. over time. The high- $a$ investors (hence low elasticity of intertemporal substitution] prefers lower consumption growth than the low-a investors. In equilibrium, the consumption of the high- $a$ investors will be less sensitive to changes in aggregate consumption than the consumption of the low-a investors. Furthermore, the high-a investors' share of aggregate consumption is higher [lower] than that of the low- $a$ investors when the level of aggregate consumption is high [low]. Thus the consumption growth of individual investors now depends on the level of aggregate consumption. Consequently, the instantaneous interest rate and the shape of the yield curve changes over time as the aggregate consumption changes.

We find that the borrowing and lending between the two classes of investors in financing their optimal consumption plans tends to increase the volatility of short-term yields. In particular, the short-term yields can move outside the range bounded by the values they would take in worlds populated only by one class of investors. On the other hand, the long-term yields with both investors present are closely related to the bounds given by the two yield curves with only one of the two classes of investors. It always approaches the lower bound as the maturity increases. Under mild growth of the economy, the preferences of the high-a investors dominate the long-term yields even though the low- $a$ investors may eventually own the whole economy. This is independent of the current wealth distribution between the two investors. This result implies that investors with small relative wealth can have large effects on bond yields. Note 
that the more risk-averse investors are more averse against low levels of future consumption. The long-term bonds are more attractive to them as hedging instruments against future down turns of the economy. Consequently, the high- $a$ investors can exert stronger influence on the equilibrium prices of long-term bonds when the probability of future down turns is not too small, i.e., the growth of the economy is not too high.

A close cousin of the current model is Dumas (1989). He considers the equilibrium of a production economy with two investors, one of whom has logarithmic preferences and the other power preferences. Since the growth of the economy is endogenously determined in a production economy, Dumas has to conjecture the existence of equilibrium and resorts to numerical solutions in his analysis. The pure exchange economy considered here allows closed-form solutions to the equilibrium which enables us to analyze the behavior of bond yields. The current model is also related to the international growth models with heterogeneous agents [see, e.g., Solow (1956), Cass (1965), Koopmans (1965), Becker (1978), Lucas and Stokey (1984)]. These models often assume certainty, hence are less interesting for studying the term structure of interest rates.

The paper is organized as follows. In section 2, we define the basic model where there are two classes of investors and the aggregate endowment follows a simple geometric Brownian motion. The equilibrium of the economy is derived in section 3 . In section 4 , we calculate the equilibrium bond prices and analyze the effect of preference heterogeneity on the behavior of bond yields. Extensions of the basic model to allow more than two classes of investors and more general processes of the aggregate endowment are discussed in section 5 . Some further comments are given in Section 6. All proofs are in the appendix.

\section{The Basic Model}

We consider a pure exchange economy of a single perishable consumption good [the numeraire]. The economy is endowed with a flow of the consumption good. Let the rate of endowment flow be $Y_{t}$ at $t$ for $t \in[0, T]$ which follows a geometric Brownian motion:

$$
d Y_{t}=\mu Y_{t} d t+\sigma Y_{t} d w_{t}, \quad t \in[0, T]
$$

where $Y_{0}>0, \mu \geq 0$ and $\sigma>0$ are constants, and $w_{t}$ is a standard Wiener process. [Throughout the paper, equalities or inequalities involving random variables are always in the sense of almost surely with respect to the underlying probability measure.] The process $Y_{t}$ has a natural 
boundary at 0 which is attractive when $\mu<\frac{1}{2} \sigma^{2}$ but always unattainable [see Karlin and Taylor (1981)]. This implies that $Y_{t}$ is strictly positive with probability $1 . Y_{t}$ as defined by (1) has the following solution:

$$
Y_{t}=Y_{0} \exp \left\{\left(\mu-\frac{1}{2} \sigma^{2}\right) t+\sigma \int_{0}^{t} d w_{s}\right\}
$$

Conditional on $Y_{t}, Y_{t+\tau}$ is log-normally distributed. Define $g_{t}(\tau) \equiv Y_{t+\tau} / Y_{t}$ as the [gross] growth rate of aggregate consumption. We have $\mathrm{E}\left[\log g_{t}(\tau)\right]=\left(\mu-\frac{1}{2} \sigma^{2}\right) \tau$ and $\operatorname{Var}\left[\log g_{t}(\tau)\right]=\sigma^{2} \tau$.

There exists a market where shares of the aggregate endowment (the "stock") are traded. Holding one share of the stock from $t=0$ to $t=T$ yields the payoff [i.e., the dividend] at rate $\left\{Y_{t}, t \in[0, T]\right\}$. In addition, there exists a "money market" where a locally risk-free security can be traded [i.e., investors can borrow from or lend to each other without default]. For $t \in[0, T]$, let $S_{t}$ be the price of the stock [ex-dividend] and $r_{t}$ the instantaneous interest rate.

Investors in the economy can trade competitively in the securities market and consume the proceeds. Let $c_{t}$ be an investor's consumption rate at $t, \alpha_{t}$ his holdings of the risk-free asset and $\theta_{t}$ his holdings of the stock. The consumption and trading strategies $\left\{c_{t},\left(\alpha_{t}, \theta_{t}\right)\right\}$ are adopted processes satisfying the standard integrability conditions:

$$
\int_{0}^{T} c_{t}^{2} d t<\infty, \quad \int_{0}^{T}\left|\alpha_{t} r_{t} d t+\theta_{t}\left(Y_{t} d t+d S_{t}\right)\right|<\infty \text { and } \int_{0}^{T} \theta_{t}^{2} d\left[S_{t}\right]<\infty
$$

where $\left[S_{t}\right]$ denotes the quadratic variation process of $S_{t} \cdot{ }^{3}$ The investor's wealth process defined by $W_{t} \equiv \alpha_{t}+\theta_{t} S_{t}$ must (a) be positive with probability one, and (b) conform to the stochastic differential equation

$$
d W_{t}=\alpha_{t} r_{t} d t+\theta_{t}\left(Y_{t} d t+d S_{t}\right)-c_{t} d t
$$

The restriction of positive wealth is to rule out arbitrage opportunities [following Dybvig and Huang (1988)]. Let $\Theta$ denote the set of trading strategies that satisfy the above conditions.

There are two classes of identical investors in the economy, denoted as 1 and 2 . Both classes of investors are initially endowed with only shares of the stock. Let $\alpha_{i, 0_{-}}$and $\theta_{i, 0_{-}}$be the initial shares of the risk-free security and the stock of class- $i$ investors. Then $\alpha_{i, 0_{-}}=0, \theta_{i, 0_{-}}>0$, $i=1,2$, and $\sum_{i=1}^{2} \theta_{i, 0_{-}}=1$. Note that $\left(\alpha_{i, 0}, \theta_{i, 0}\right)$ which denotes the optimal holdings of class- $i$

\footnotetext{
${ }^{3}$ See, e.g., Karatzas and Shreve (1988) for a discussion on the quadratic variation process of a given process.
} 
investors is in general different from their endowment $\left(0, \theta_{i, 0_{-}}\right)$. A class- $i$ investor, $i=1,2$, chooses his consumption/trading strategy $\left\{c_{i},\left(\alpha_{i}, \theta_{i}\right)\right\}$ to maximize his lifetime expected utility

$$
\mathrm{E}_{\mathrm{t}}\left[\int_{t}^{T} e^{-\rho(s-t)} \frac{c_{i, s}^{1-a_{i}}-1}{1-a_{i}} d s\right], \quad a_{i}>0,
$$

where $\rho>0$ is the time discount parameter which is the same across investors. Since investors within each class are identical, we do not distinguish them and simply denote them respectively as investor $i, i=1,2$. Both classes of investors have constant relative risk aversion (CRRA). Throughout sections $3-4$, it is further assumed that $a_{1}=1$ and $a_{1}=1 / 2$ to obtain simple solutions [the utility function with $a=1$ is obtained by taking the $\operatorname{limit}_{\lim } \rightarrow 1 \frac{c^{1-a}-1}{1-a}=\log c$ ]. Thus, class-1 investors have logarithmic utility function and class- 2 investors have square-root utility function, and class- 1 investors are more risk averse than class-2 investors [in terms of relative risk aversion]. In section 5 , we will consider relaxing this restrictive assumption.

In addition, we impose the following growth condition on the parameter values:

$$
\rho>\frac{1}{2} \max \left[0, \mu-\frac{1}{4} \sigma^{2}\right] \text {. }
$$

This growth condition guarantees that investors' expected utilities are uniformly bounded for all $T \in[0, \infty)$ given the aggregate consumption process in (1). This allows us to take the limit $T \rightarrow \infty$ in our future discussions.

Before we consider the equilibrium of the economy as defined above, a few comments on the economy are in order. For simplicity in exposition, $Y_{t}$ is restricted to be a univariate diffusion process with linear drift and diffusion coefficient. Extensions to the multivariate case will be considered in section 5. Extensions to more general forms for the drift and the diffusion coefficient including path dependence are also possible.

In specifying the securities market, we have only introduced the stock and the locally riskfree security as traded securities. As will be shown later, given the current process of $Y_{t}$, the stock and the risk-free security are sufficient to dynamically complete the securities market in the sense of Harrison and Kreps (1979). Arbitrary consumption plans [satisfying certain integrability conditions as specified later] can be financed by continuously trading in the stock and the riskfree security. Allowing additional securities will not affect the nature of the equilibrium. Thus, in deriving the market equilibrium we will consider the securities market as consists of only the stock and the risk-free security. Simple arbitrage arguments can then be used to price other securities if they exist. 
We have assumed that there are only two classes of investors in the economy and they behave competitively in the market. Since investors within each class have the same isoelastic preferences, we can represent each class with a single representative investor who has same preferences as the individual investors and the total endowment of the class [see, e.g., Rubinstein (1974)]. In deriving the equilibrium, we can then treat the economy as populated with the two representative investors for the two classes and they behave competitively. In the remainder of the paper, we will treat the two representative investors as two individual investors without referring to the class of investors they represent and denote them as investor 1 and 2 , respectively.

\section{Market Equilibrium}

In this section, let us consider the market equilibrium of the economy defined above. We first derive a solution to the market equilibrium and then discuss the general nature of the equilibrium and the pricing implications.

\subsection{Deriving the Equilibrium}

The definition of a market equilibrium follows Radner (1972):

Definition $1 A$ market equilibrium of the economy is the pair of price process $\{S, r\}$ and consumption-trading strategies $\left\{c_{i},\left(\alpha_{i}, \theta_{i}\right) ; i=1,2\right\}$ such that (i) $\left\{c_{i},\left(\alpha_{i}, \theta_{i}\right)\right\}, i=1,2$, maximizes investor $i$ 's expected utility:

$$
\begin{array}{cl}
\sup _{\substack{\left.c_{i},\left(\alpha_{i}, \theta_{i}\right)\right\} \\
\text { s.t. }}} & \mathrm{E}_{\mathrm{t}}\left[\int_{t}^{T} e^{-\rho(s-t)} \frac{c_{i, s}^{1-a_{i}}-1}{1-a_{i}} d s\right], \quad t \in[0, T] \\
& d W_{i, t}=\alpha_{i, t} r_{t} d t+\theta_{i, t}\left(Y_{t} d t+d S_{t}\right)-c_{i, t} d t
\end{array}
$$

and (ii) markets clear:

$$
\sum_{i=1}^{2} \theta_{i, t}=1, \quad \sum_{i=1}^{2} \alpha_{i, t}=0 .
$$

Here, $W_{i, 0}=\theta_{i, 0_{-}} S_{0}$.

Equation (7) gives the market-clearing of the securities market. The market-clearing of the goods market is guaranteed by Walras' law. Combining the two market clearing conditions in (7), we have $W_{1, t}+W_{2, t}=S_{t}$. 
The equilibrium is derived in three steps. First, we solve the Pareto-optimal allocations of the economy. Next, it is shown that each Pareto-optimal allocation can be supported by an Arrow-Debreu equilibrium where investors can trade arbitrary future payoff streams at the initial date and achieve the given allocation. Finally, we construct the dynamic implementation of the Arrow-Debreu equilibrium [see Duffie and Huang (1985)] where investors continuously trade the stock and the risk-free security at prices given by the pricing functional in the ArrowDebreu equilibrium and achieve the same allocation in equilibrium. This then gives the market equilibrium of the economy. ${ }^{4}$

When both investors have positive initial wealth, an allocation $\left\{c_{1}, c_{2}\right\}$ is Pareto-optimal if and only if there is a constant $\lambda \in(0,1)$ such that $\left\{c_{1}, c_{2}\right\}$ solves the problem

$$
\begin{array}{ll}
\sup _{\left\{c_{1}, c_{2}\right\}} & \mathrm{E}_{0}\left\{\int_{0}^{T} e^{-\rho t}\left[\lambda \log c_{1, t}+2(1-\lambda) \sqrt{c_{2, t}}\right] d t\right\} \\
\text { s.t. } & c_{1, t}+c_{2, t} \leq Y_{t}, \quad t \in[0, T] .
\end{array}
$$

Here, $\lambda$ is the weight of the log-investor's utility in the welfare function to be maximized. Note that in an exchange economy, there is no intertemporal transformation of resources. The intertemporal resource constraint in (8) is simply the collection of resource constraints for each date and each state. Furthermore, the investors' preferences are time-additive and state-separable, and so is the welfare function. Thus maximizing the expected intertemporal welfare function in (8) is equivalent to maximizing the welfare function period by period and state by state subject to the corresponding resource constraint. For each period and each state, the maximization problem takes the following form:

$$
\sup _{c_{1}+c_{2} \leq Y} e^{-\rho t}\left[\lambda \log c_{1}+2(1-\lambda) \sqrt{c_{2}}\right] .
$$

Its solution gives the optimal sharing rule between the two investors.

Lemma 1 Given $\lambda \in(0,1)$, the optimal sharing rule between the two investors is

$$
\hat{c}_{1}(Y, \lambda)=\frac{1}{2}\left(\frac{\lambda}{1-\lambda}\right)^{2}\left[\sqrt{1+4\left(\frac{1-\lambda}{\lambda}\right)^{2} Y}-1\right], \quad \hat{c}_{2}(Y, \lambda)=Y_{t}-\widehat{c}_{1}(Y, \lambda) .
$$

\footnotetext{
${ }^{4}$ Many authors have studied the existence of market equilibrium in quite general settings in continuous-time [see, e.g., Duffie and Zame (1991), Mas-Colell and Zame (1991), Karatzas, Lehoczky and Shreve (1990)]. However, the model defined above does not directly fit into their framework. In particular, the aggregate endowment process as specified by (1) is not bounded away from zero which is often assumed in the literature.
} 
Also, $\lambda \widehat{c}_{1}(Y, \lambda)^{-1}=(1-\lambda) \widehat{c}_{2}(Y, \lambda)^{-1 / 2}$.

The optimal sharing rule as a function of $Y$ is non-linear and only depends on $\lambda$. Furthermore, in a Pareto-optimum the marginal utilities of the two investors are linearly related. Given $\lambda$, the Pareto-optimal allocation is simply $\widehat{c}_{i, t}=\widehat{c}_{i}\left(Y_{t}, \lambda\right), i=1,2, \forall Y_{t}$ and $t \in[0, T]$. For future convenience, define a representative agent by his utility function at $t$ over aggregate consumption $Y_{t}$ as follows: $u_{\lambda}\left(Y_{t}, t\right) \equiv e^{-\rho t} u_{\lambda}\left(Y_{t}\right)$ and

$$
u_{\lambda}\left(Y_{t}\right) \equiv\left[\lambda \log \widehat{c}_{1}\left(Y_{t}, \lambda\right)+2(1-\lambda) \sqrt{\hat{c}_{2}\left(Y_{t}, \lambda\right)}\right]
$$

For simplicity in notation, we have used $u_{\lambda}$ for the both the time discounted and the undiscounted utility function of the representative agent. Let $b \equiv 4(1-\lambda)^{2} / \lambda^{2}$. The marginal utility of the representative agent over aggregate consumption is

$$
m_{t} \equiv \frac{\partial u_{\lambda}\left(Y_{t}, t\right)}{\partial Y_{t}}=\left(\frac{b}{2+\sqrt{b}}\right) \frac{e^{-\rho t}}{\sqrt{1+b Y_{t}}-1}, \quad t \in[0, T]
$$

It is easy to see that the relative marginal utility of the representative agent [between any two states] are the same as the relative marginal utilities of the two individual investors.

For any Pareto-optimal allocation, let us now derive the Arrow-Debreu equilibrium that supports the allocation. In an Arrow-Debreu equilibrium, investors can trade arbitrary payoff streams at the initial date. The equilibrium is defined as the pricing function $\left\{\phi_{0, s}, s \in[0, T]\right\}$, such that the price of an arbitrary payoff stream $\left\{X_{s}, s \in[0, T]\right\}$ at $t=0$ is given by the linear functional $\Phi_{0}(X)=\mathrm{E}_{0}\left[\int_{0}^{T} \phi_{0, s} X_{s} d s\right]$, and the market clears. Given the specific form of investor preferences assumed here, we have the following lemma:

Lemma 2 Given $\lambda \in(0,1)$ and the corresponding optimal allocation $\left(\widehat{c}_{1}, \hat{c}_{2}\right)$, there exists an Arrow-Debreu equilibrium that leads to the same allocation where the pricing function is given by $\phi_{0, s}=m_{s} / m_{0}, s \in[0, T] .^{5}$

Clearly, the pricing function $\phi_{0, s}$ is positive. The value of the pricing function for any state at $s$ is simply the ratio between the marginal utility of the representative agent in that state and his marginal utility at 0 . In general, $\phi_{0, s}$ may depend on $s$ and the whole time path of $Y_{s}$ up to $s$, which gives the complete description of the underlying state of the economy at $s$.

\footnotetext{
${ }^{5}$ In a setting more general than the current one, Araujo and Monteiro (1989) have shown that the Second Welfare Theorem holds [see also Duffie and Zame (1989) and Mas-Colell and Zame (1991)].
} 
In the current setting, however, due to the time-additive and state-separable preferences of the investors, it only depends on $s, Y_{s}, Y_{0}$ and $\lambda$. Thus we can write $\phi_{0, s}=\phi\left(Y_{s}, s ; Y_{0} ; \lambda\right)$. Although $\phi_{0, s}$ gives the pricing function at the initial date, one can also derive the pricing function for any future time. The price function at $t$ is simply $\phi_{t, s}=m_{s} / m_{t}$ where $t, s \in[0, T]$ and $s \geq t$. Clearly, $\phi_{t, s}=\phi\left(Y_{s}, s ; Y_{t}, t ; \lambda\right)$. The Arrow-Debreu price of payoff $\left\{X_{s}, s \in[0, T]\right\}$ at $t$ is then $\Phi_{t}(X)=\mathrm{E}_{\mathrm{t}}\left[\int_{t}^{T} \phi_{t, s} X_{s} d s\right]$.

In the literature, it is often assumed that the pricing function $\phi_{t}$ is bounded above and away from zero [see, e.g., Duffie and Huang (1985), Duffie (1986), Huang (1987), Duffie and Zame (1989)]. These conditions are not satisfied here. This implies that securities with payoffs satisfying simple integrability conditions such as $\mathrm{E}_{0}\left[\int_{0}^{T} X_{s}^{2} d s\right]<\infty$ do not always have finite prices. This is not surprising when the state prices are unbounded. Securities that have nontrivial payoffs in states with high state prices will certainly have high prices at time 0 . In the remainder of the paper, we will restrict to securities that have finite Arrow-Debreu prices.

Let us now consider the market equilibrium as defined at the beginning of this section. Duffie and Huang (1985) have shown in a quite general setting that for any Arrow-Debreu equilibrium, a corresponding market equilibrium can be constructed as its dynamic implementation to achieve the same allocation. Unfortunately, the current model does not meet some of the regularity conditions required by their results. However, by slightly modifying their approach we can derive the dynamic equilibrium as follows. Given an Arrow-Debreu equilibrium as specified in lemma 2, the stock prices and interest rates are first calculated using the Arrow-Debreu pricing function. It is then shown that budget-feasible trading strategies can be found for each individual investor to finance his consumption plan given in the Arrow-Debreu equilibrium. Finally, it is shown that the above consumption/trading strategy for each investor is optimal since any trading strategy that gives higher expected utility is not budget-feasible [see the appendix for a formal proof].

Lemma 3 Given an Arrow-Debreu equilibrium as defined in lemma 2, there exists a dynamic implementation in which prices of traded securities are given by

$$
S_{t}=\mathrm{E}_{\mathrm{t}}\left[\int_{t}^{T}\left(\frac{m_{s}}{m_{t}}\right) Y_{s} d s\right], \quad r_{t}=-\frac{\mathrm{E}_{\mathrm{t}}\left[d m_{t}\right]}{m_{t} d t}, \quad t \in[0, T],
$$

investors optimally choose the consumption plan $\left(\hat{c}_{1}, \hat{c}_{2}\right)$ financed respectively by budget-feasible trading strategies, and the securities market clears. 
Given the definition of $m_{t}$ and the process for $Y_{t}, S_{t}$ and $r_{t}$ can be expressed as functions of $Y_{t}$, $t$ and $\lambda$. Thus, we can write $S_{t}=S\left(Y_{t}, t ; \lambda\right)$ and $r_{t}=r\left(Y_{t}, t ; \lambda\right)$.

Combining lemmas $1-3$, we obtain the solution to the market equilibrium as summarized in the following theorem:

Theorem 1 For the economy defined in Section 2, there exists a market equilibrium in which (i) the equilibrium prices of traded securities are given by (12); (ii) investors' optimal consumption strategies are

$$
\widehat{c}_{1, t}=\frac{2}{b}\left[\sqrt{1+b Y_{t}}-1\right], \quad \widehat{c}_{2, t}=Y_{t}-\widehat{c}_{1, t}
$$

which are financed, respectively, by the following trading strategies

$$
\begin{aligned}
& \hat{\alpha}_{1, t}=\frac{2}{b \rho}\left[1-e^{-\rho(T-t)}\right]\left(\sqrt{1+b Y_{t}}-1\right)-\hat{\theta}_{1, t} S_{t}, \quad \hat{\theta}_{1, t}=\frac{b}{\rho} \frac{1-e^{-\rho(T-t)}}{S_{Y} \sqrt{1+b Y_{t}}} \\
& \hat{\alpha}_{2, t}=-\widehat{\alpha}_{1, t}, \quad \widehat{\theta}_{2, t}=1-\widehat{\theta}_{1, t}
\end{aligned}
$$

where $S_{Y}=\frac{\partial S}{\partial Y}$; and (iii) $b$ is determined by

$$
\theta_{1,0_{-}} \mathrm{E}_{0}\left[\int_{0}^{T} e^{-\rho \tau}\left(\sqrt{1+b Y_{0} g_{t}(\tau)}+1\right) d \tau\right]=\frac{2}{\rho}\left(1-e^{-\rho T}\right) .
$$

Furthermore, $S_{Y}>0$.

Note that multiplying $1 /\left(b m_{0}\right)$ to the two sides of $(14)$, we have $W_{1,0}=\theta_{1,0_{-}} S_{0}$ for the left-hand side, which is investor 1's initial wealth, and $E_{0}\left[\int_{0}^{T} \phi_{0, t} \hat{c}_{1, t} d t\right]$ for the right-hand side, which is the cost of his optimal consumption plan. Thus (14) is simply investor 1's budget constraint, which uniquely determines $b$ (or $\lambda$ ) in terms of the initial condition of the economy, $\theta_{1,0_{-}}$and $Y_{0}{ }^{6}$

\subsection{Properties of the Equilibrium}

Given that the uncertainty of the economy is completely characterized by the process of aggregate consumption $Y_{t}$ which is a univariate diffusion, the stock and the [locally] risk-free security allow the market to be dynamically complete. Any consumption patterns [that have finite ArrowDebreu prices] can be financed by continuous trading in these two securities. Thus investors are able to achieve Pareto-optimal allocations in the market equilibrium. Introducing other

\footnotetext{
${ }^{6}$ Since $W_{1,0}+W_{2,0}=S_{0}=S\left(Y_{0}, 0 ; \lambda\right)$, we can also express $\lambda$ in terms of the two investors' initial wealth.
} 
securities will not change the equilibrium allocations. Furthermore, any other securities can be synthesized by trading only in the stock and the risk-free security. Their prices should equal the cost of the synthesizing strategy. As we will see in section 5 when $Y_{t}$ follows more general processes, more securities will be needed to complete the market.

In deriving the equilibrium, we have introduced $\lambda$, the relative weight of the two investors in the welfare function, to characterize the Pareto-optimal allocations and the supporting equilibria. Equation (14) uniquely determines $\lambda$ (or $b$ ) in terms of $\theta_{1,0_{-}}$and $Y_{0}$. It is easy to show that $\lambda$ is an increasing function of $\theta_{1,0_{-}}$[holding $Y_{0}$ constant] and a decreasing function of $Y_{0}$ [holding $\theta_{1,0_{-}}$constant]. When $\theta_{1,0_{-}} \rightarrow 1, \lambda \rightarrow 1, \hat{c}_{1}(Y, \lambda) \rightarrow Y$ and $\hat{c}_{2}(Y, \lambda) \rightarrow 0$. This is the limiting case when the economy is populated only by investor 1 . When $\theta_{1,0_{-}} \rightarrow 0, \lambda \rightarrow 0, \widehat{c}_{1}(Y, \lambda) \rightarrow 0$ and $\hat{c}_{2}(Y, \lambda) \rightarrow Y$. This is the limiting case when the economy is populated only by investor 2. It is also interesting to consider the allocation of consumption when the initial aggregate endowment is very low or very high, i.e., when $Y_{0} \rightarrow 0$ or $Y \rightarrow \infty$ [holding $\theta_{1,0_{-}}$constant]. It is easy to show that when $Y_{0} \rightarrow 0, \lambda \rightarrow 1, \widehat{c}_{1}(Y, \lambda) \rightarrow Y$ and $\widehat{c}_{2}(Y, \lambda) \rightarrow 0$. When $Y_{0} \rightarrow \infty, \lambda \rightarrow 0$, $\widehat{c}_{1}(Y, \lambda) \rightarrow 0$ and $\widehat{c}_{2}(Y, \lambda) \rightarrow Y$. This suggests that $\lambda$ does not simply represent the relative wealth of the two investors, even though it can be expressed as a function of the wealth of the two investors. For example, even when $\theta_{1,0_{-}} \gg \theta_{1,0_{-}}>0$ [thus $W_{1,0} / W_{2,0} \gg 1$ ], $\lambda$ can be very small if $Y_{0}$ is very large. In other words, $\lambda$ not only depends on the initial relative wealth of the two investors, but also depends on the level of total initial wealth.

It is important to note that $\lambda$ depends only on the initial conditions of the economy, and remains constant afterwards. Given the initial condition of the economy [i.e., $Y_{0}$ and $\theta_{1,0_{-}}$], $Y_{t}$ completely determines the state of the economy at $t$. As the economy evolves, the state of the economy, security prices, investors' wealth and their security holdings do change. But the sharing rule does not. The intuition behind this result is simple. In the current setting, the securities market is dynamically complete. In equilibrium, investors follow optimal trading strategies to achieve consumption distributions such that the relative marginal utilities [for any two states] are equal for all investors. [Otherwise, gains could be made for the investors by deviating from their optimal trading strategies.] For example, if at the current level of consumption, investor 1 's marginal utility is more sensitive to changes in the level of consumption than investor 2. Investor 1 will then optimally hold a portfolio that yields lower [higher] returns than the portfolio of investor 2 when aggregate consumption increases [decreases]. Consequently, their marginal 
utility remains proportional independent of future changes in aggregate consumption. This implies that in all states, the two investors' marginal utilities are linearly related with a constant proportionality constant. This condition then gives the sharing rule between the two investors, which does not change over time. ${ }^{7}$

Even though the sharing rule between the two investors does not change over time, the actual consumption of the two investors does change as the aggregate consumption $Y_{t}$ changes. For example, as $Y_{t}$ increases, investor 1's percentage share in aggregate consumption decreases and investor 2's share increases. When $Y_{t}$ drifts to zero, investor 1's percentage share in aggregate consumption drifts to one. On the other hand, when $Y_{t}$ drifts to infinity, investor 1 's percentage share in aggregate consumption drifts to zero. This result is quite intuitive given the investors preferences. At low [high] levels of consumption, investor 1's marginal utility is higher [lower] than investor 2's marginal utility. In equilibrium, investor 1 maintains higher [lower] level of consumption than investor 2 when the aggregate consumption is low [high]. As investors' consumption changes, security prices also change.

Investors' optimal consumption policies are financed by their corresponding trading strategies. From theorem 1 , we have the following the corollary.

Corollary 1 For $\lambda \in(0,1), \alpha_{1, t}>0$ and $\alpha_{2, t}<0$. When $Y_{t} \rightarrow 0, \theta_{1, t} S_{t} / W_{1, t} \rightarrow 1, W_{1, t} / S_{t} \rightarrow 1$ and $W_{2, t} / S_{t} \rightarrow 0$. When $Y_{t} \rightarrow \infty, \theta_{1, t} S_{t} / W_{1, t} \rightarrow 1 / 2, W_{1, t} / S_{t} \rightarrow 0$ and $W_{2, t} / S_{t} \rightarrow 1$.

Thus, investor 1 is the lender and investor 2 is the borrower. This is not surprising given that investor 1 is more risk-averse than investor 2. Furthermore, investor 1 shifts his portfolio toward the stock [the risk-free security] when the stock price drops [arises] while investor 2 does the opposite. Also, investor 1's relative wealth approaches 1 and 0 as the level of aggregate consumption shifts to 0 and $\infty$, respectively. This implies that investor 1 follows a strategy that pays off in bad states of the economy since his marginal utility is higher than that of investor 2 at low levels of consumption.

\footnotetext{
${ }^{7}$ As also implied by the above discussion, if one re-calculate the equilibrium at a later date, the same $\lambda$ will be obtained. As the aggregate endowment changes, investors' wealth also changes. But $\lambda$ as a function of both investors' wealth remains constant.
} 


\subsection{Security Prices in Equilibrium}

In the market equilibrium, we can also price securities that can be replicated by dynamic trading strategies at finite costs. Suppose a security has payoff $\left\{X_{s}, s \in[t, T]\right\}[t \geq 0]$, its price is

$$
P_{t}=\mathrm{E}_{\mathrm{t}}\left[\int_{t}^{T}\left(\frac{m_{t+s}}{m_{t}}\right) X_{s} d s\right]=\mathrm{E}_{\mathrm{t}}\left[\int_{t}^{T} e^{-\rho(s-t)} \frac{\sqrt{1+b Y_{t}}-1}{\sqrt{1+b Y_{s}}-1} X_{s} d s\right]
$$

If $X_{t}$ only depends on $Y_{t}$ and $t$, its price $P_{t}$ as a function of $Y$ and $t$ satisfies the stochastic equation:

$$
d P=\mu_{P} P d t+\sigma_{P} P d w
$$

where $\mu_{P}=\left(\frac{\partial P}{\partial t}+\mu Y \frac{\partial P}{\partial Y}+\frac{1}{2} \sigma^{2} Y^{2} \frac{\partial^{2} P}{\partial Y^{2}}\right) / P$ and $\sigma_{P}=\sigma Y P_{Y} / P$. [Here, it is assumed that $X=f(Y, t)$ is twice differentiable with respect to $Y$ ]. From (15), we obtain the following partial differential equation for $P$ :

$$
\frac{\partial P}{\partial t}+\mu Y \frac{\partial P}{\partial Y}+\frac{1}{2} \sigma^{2} Y^{2} \frac{\partial^{2} P}{\partial Y^{2}}-r P+X=\pi \sigma Y \frac{\partial P}{\partial Y}
$$

where $r$ is given by (12) and

$$
\pi \equiv-\sigma \frac{Y}{m} \frac{\partial m}{\partial Y}=\frac{b \sigma Y}{2 \sqrt{1+b Y}(\sqrt{1+b Y}-1)} .
$$

Given $X_{t}$ and proper boundary conditions, the solution to (16) gives the equilibrium price of the security. 8

The variable $\pi$ can be interpreted as the market price of risk. Rewrite (16) as

$$
\frac{\mu_{P}+X / P-r}{\sigma_{P}}=\pi
$$

The left hand side is simply the Sharpe measure of the security which is the expected excess return on the security normalized by its standard deviation. Given that there is only one source of risk in the current situation, the Sharpe measure is the same for all risky securities and we can call it the market price of risk. Since $m_{t}$ is simply the marginal utility of the representative agent at time $t$, we can rewrite (17) as

$$
\pi_{t}=\left[-\frac{Y_{t} u_{\lambda}^{\prime \prime}\left(Y_{t}\right)}{u_{\lambda}^{\prime}\left(Y_{t}\right)}\right] \sigma \equiv a_{\lambda}\left(Y_{t}\right) \sigma
$$

\footnotetext{
${ }^{8}$ For a general framework of intertemporal asset pricing based on investor optimality conditions, see Merton (1973) and Cox, Ingersoll and Ross (1985b).
} 
where $a_{\lambda}(Y)$ is the relative risk-aversion of the representative agent at consumption level $Y$. Thus, the market price of risk is proportional to the uncertainty in consumption growth $\sigma$ and the proportionality constant is just the relative risk-aversion of the representative agent. As $Y_{t}$ changes over time, the risk-aversion of the representative agent also changes and so does the market price of risk. It can be shown that $a_{\lambda}\left(Y_{t}\right)$ monotonically decreases with $Y_{t}$ and lies in the interval $\left(a_{2}, a_{1}\right)=(1 / 2,1)$. It approaches $a_{1}$ and $a_{2}$ as $Y_{t}$ approaches 0 and $\infty$ respectively. Note that the market price of risk when only investor $i, i=1,2$, is present is simply $\pi^{(i)}=a_{i} \sigma$ which is constant. Thus, the market price of risk when both investors are present lies between $\pi^{(2)}$ and $\pi^{(1)}$ and varies over time.

Given the growth condition (5), the stock price and bond prices are well defined when we take the limit $T \rightarrow \infty$. As a matter of fact, the limiting economy and its equilibrium as the limit of the equilibrium with finite $T$ are well defined. ${ }^{9}$ For simplicity in exposition, in the remainder of this paper, we will consider the limiting economy and its equilibrium when $T \rightarrow \infty$. In this case, the economy has an infinite horizon, hence the state of the economy at time $t$ only depends on the level of aggregate consumption then given the initial condition of the economy, not on $t$ itself.

\section{Bond Prices and Yields}

Let us now consider the equilibrium term structure of interest rates. Let $B_{t}(\tau)$ be the price of a pure discount bond at $t$ that matures at $t+\tau$ where $t, \tau>0$. Its payoff process is $X_{s}^{B}=\delta(s-t-\tau)$ where $\delta(\cdot)$ is the Dirac $\delta$-function. Substituting $X_{s}^{B}$ into the pricing equation (15), we have the following expression for its price at $t$ :

$$
B_{t}(\tau)=e^{-\rho \tau} \mathrm{E}_{\mathrm{t}}\left[\frac{\sqrt{1+Y_{t}}-1}{\sqrt{1+Y_{t} g_{t}(\tau)}-1}\right]
$$

Its yield to maturity $y_{t}(\tau)$ is defined by $y_{t}(\tau) \equiv-\frac{1}{\tau} \log B_{t}(\tau)$. How the bond yield changes with maturity gives the term structure of interest rates. Since the state of the economy at $t$ depends on $Y_{t}$, the bond prices and the term structure will also depend on $Y_{t}$. As $Y_{t}$ changes over time, the term structure also changes.

\footnotetext{
${ }^{9}$ Certain technical modifications are needed in analyzing the infinite-horizon counterpart of a finite-horizon economy. See Huang and Pagès (1990) for more detailed discussions. It is easy to see that those modifications are quite straightforward in the current setting.
} 


\subsection{Limiting Cases}

Before we consider the bond prices and the term structure of interest rates when both investors are present, let us first examine the limiting cases when only one of the two investors is present in the economy [i.e., when $\theta_{1,0_{-}} \rightarrow 1$ or 0 ]. Our model in the two limiting cases is similar to CIR, except that the specific process of aggregate consumption is different. ${ }^{10}$ The resulting interest rate process is identical to the one analyzed by Vasicek (1977) in a partial equilibrium context and Stapleton and Subrahmanyam (1990), McCulloch (1993) in a general equilibrium context.

Let $B_{t}^{(i)}(\tau)$ be the price of a pure discount bond at $t$ with maturity $\tau$ when only investor $i$ is present in the economy. $B_{t}^{(i)}(\tau)$ can be calculated from (19) by properly taking the limits: $b \rightarrow 0$ for $i=1$ and $b \rightarrow \infty$ for $i=2$ respectively. ${ }^{11}$ We have

$$
B_{t}^{(i)}(\tau)=e^{-\rho \tau} \mathrm{E}_{\mathrm{t}}\left[g_{t}(\tau)^{-a_{i}}\right], \quad i=1,2
$$

In both of these two limiting cases, the bond prices do not depend on the current level of aggregate endowment. They only depend on the expectations of future growth rates. This result is well known [see, for example, Cox, Ingersoll and Ross (1985a), Dumas (1989) and Stapleton and Subrahmanyam (1990)]. From the distributional assumptions about the growth rates, the following expressions are obtained for bond prices in the two limiting cases:

Lemma 4 Given the process of $Y_{t}$, we have

$$
B_{t}^{(i)}(\tau)=e^{-\tau^{(i)} \tau}
$$

where $r^{(i)}=\rho+a_{i}\left(\mu-\frac{1+a_{i}}{2} \sigma^{2}\right)$ and $i=1,2$.

It is clear that in the two limiting cases the interest rate is constant over time and the term structure is flat, i.e., $y_{t}^{(i)}(\tau)=r^{(i)}, \forall t$. Even though the aggregate consumption $Y_{t}$ varies over time, the yield curve stays constant.

\footnotetext{
${ }^{10} \mathrm{CIR}(1985 \mathrm{a})$ consider a production economy where the aggregate consumption is endogenously determined by the representative investor's optimal consumption-trading strategies. Sun (1992) shows that an exchange economy can be constructed which is analogous to the CIR production economy. The endowment process in the exchange economy is taken to be the same as the optimal consumption process in the CIR model. The pricing implications are the same for the two economies.

${ }^{11}$ In obtaining the bond prices in the two limiting cases, the limit $b \rightarrow 0$ or $b \rightarrow \infty$ is taken under the integration. The order of taking the limit and integration is irrelevant here. This can be easily shown by applying standard convergence results.
} 


\subsection{Bond Prices with Two Investors}

We now consider the bond prices and yields when both investors are present in the economy. For simplicity in exposition, we let $b=1$ in the pricing equation (15) from now on. This implies that the following weights are assigned to the two investors in the welfare function: $\lambda=2 / 3$ and $1-\lambda=1 / 3$. As discussed in section 3.1, this choice of $\lambda$ involves certain choices of the initial condition of the economy. The qualitative behavior of bond prices and yields does not depend on this particular choice of the initial condition. Extending our analysis to the general case of $\lambda \in(0,1)$ is trivial. ${ }^{12}$ Given the initial conditions of the economy, the bond prices are completely determined by the current level of aggregate consumption.

The equilibrium bond prices can be calculated by computing the conditional expectation in (19). The results are summarized in the following theorem.

Theorem 2 When both investors are present in the economy, the equilibrium prices are given by

$$
B_{t}(\tau)=e^{-\rho \tau}\left(\sqrt{1+Y_{t}}-1\right)\left[I_{1, t}(\tau)+I_{2, t}(\tau)\right]
$$

where

$$
I_{1, t}(\tau)=\frac{1}{Y_{t}} \mathrm{E}_{\mathrm{t}}\left[g_{t}(\tau)^{-1}\right], \quad I_{2, t}(\tau)=\mathrm{E}_{\mathrm{t}}\left[\frac{\sqrt{1+Y_{t} g_{t}(\tau)}}{Y_{t} g_{t}(\tau)}\right]
$$

Furthermore, let $\delta_{t}(\tau)=\left[\mu-\frac{1}{2} \sigma^{2} \tau+\log Y_{t}\right] /\left(\sigma^{2} \tau\right)$ and $\xi_{n, t}(\tau)=\left[n-1+\delta_{t}(\tau)\right] \sigma \sqrt{\tau}$. We have

$$
\begin{aligned}
& I_{1, t}(\tau)=e^{-\xi_{1 / 2, t}(\tau) \sigma \sqrt{\tau}} \\
& I_{2, t}(\tau)=e^{-\frac{1}{2} \delta_{t}(\tau)^{2} \sigma^{2} \tau} \sum_{n=0}^{\infty} \alpha_{n}\left\{e^{\frac{1}{2} \xi_{n, t}(\tau)^{2}} \Phi\left[-\xi_{n, t}(\tau)\right]+e^{\frac{1}{2} \xi_{1 / 2-n, t}(\tau)^{2}} \Phi\left[\xi_{1 / 2-n, t}(\tau)\right]\right\}
\end{aligned}
$$

where $\alpha_{0}=1, \alpha_{n}=(-1)^{n-1} \frac{(2 n-3) ! !}{(2 n) ! !}$ for $n \geq 1$, and $\Phi(x) \equiv \frac{1}{\sqrt{2 \pi}} \int_{-\infty}^{x} e^{-x^{\prime 2} / 2} d x^{\prime}$ is the cumulative normal distribution function. Here, $n ! !=1$ for $n \leq 0$ and $n ! !=n(n-2) ! !$ for $n>0$.

Although the bond prices are expressed in the form of infinite summation, their numerical values are easy to calculate.

\footnotetext{
${ }^{12}$ As a matter of fact, there is no loss of generality by setting $b=1$ here when both investors are present. Note that there is a one-to-one correspondence between the economy with $b \in(0,1)$ and initial aggregate endowment $Y_{0}$ and the economy with $b^{\prime}=1$ and $Y_{0}^{\prime}=b Y_{0}$.
} 
It can be shown that for a given maturity, when $Y_{t} \rightarrow 0, I_{1, t}(\tau) \gg I_{2, t}(\tau)-I_{1, t}(\tau)$. Then $I_{1, t}(\tau)+I_{2, t}(\tau) \rightarrow 2 I_{1, t}(\tau)$ and $B_{t}(\tau) \rightarrow B^{(1)}(\tau)$. On the other hand, when $Y_{t} \rightarrow \infty, I_{1, t}(\tau) \ll$ $I_{2, t}(\tau)$. Then $I_{1, t}(\tau)+I_{2, t}(\tau) \rightarrow I_{2, t}(\tau) \rightarrow\left[Y_{t} g_{t}(\tau)\right]^{-1 / 2}$ and $B_{t}(\tau) \rightarrow B^{(2)}(\tau)$.

Given the equilibrium bond prices, we can derive the equilibrium yield curve. Two yields are of particular interest. One is the instantaneous interest rate $r_{t}$, which is the limiting yield as maturity goes to zero: $r_{t} \equiv \lim _{\tau \rightarrow 0} y_{t}(\tau)$. The other is the long yield which is defined as the limiting yield as maturity goes to infinity: $y_{t}(\infty) \equiv \lim _{\tau \rightarrow \infty} y_{t}(\tau)$. They give, respectively, the two ends of the yield curve.

\subsection{Instantaneous Interest Rate}

We first consider the instantaneous interest rate $r_{t}$. It can be calculated either from theorem 2 by taking the limit $\tau \rightarrow 0$ or directly from theorem 1. From theorem 1 and applying Itô's lemma to $m_{t}=m\left(t, Y_{t}\right)$ as given in (11) [with $b=1$ ], we have the following result:

Theorem 3 When both investors are present in the economy, the instantaneous interest rate is given by

$$
r_{t}=\rho+\frac{\mu Y_{t}}{2 \sqrt{1+Y_{t}}\left(\sqrt{1+Y_{t}}-1\right)}-\frac{\sigma^{2} Y_{t}^{2}\left(3 \sqrt{1+Y_{t}}-1\right)}{8\left(1+Y_{t}\right)^{\frac{3}{2}}\left(\sqrt{1+Y_{t}}-1\right)^{2}} .
$$

Given the value of $\lambda$ determined by the initial conditions of the economy [i.e., $\theta_{1,0_{-}}$and $Y_{0}$ ], $r_{t}$ depends only on the current level of aggregate consumption $Y_{t}$ [independent on the path taken to arrive at $\left.Y_{t}\right]$.

Before we analyze the dynamics of $r_{t}$, let us examine the range within which the interest rate moves. Using a two-investor economy similar to ours but with production, Dumas (1989) conjectures that the instantaneous interest rate $r_{t}$ should always lie within the range bounded by $r^{(1)}$ and $r^{(2)}$, the values it would take in worlds populated only by one of the two investors respectively. In the pure exchange economy considered here, this is generally not the case. Note that we have $r^{(1)}=\rho+\mu-\sigma^{2}$ and $r^{(2)}=\rho+\frac{1}{2} \mu-\frac{3}{8} \sigma^{2}$. If we choose $\mu=\frac{5}{4} \sigma^{2}$, then $r^{(1)}=r^{(2)}=\rho+\frac{1}{4} \sigma^{2}$. It is easy to show that in this case, $r_{t}<r^{(1)}=r^{(2)}$ for $Y_{t} \in(0, \infty)$. $r_{t}$ reaches a unique local minimum of $\rho+\frac{25}{108} \sigma^{2}$ in the interval at $Y_{t}=5 / 4$. Thus, in the current model the interest rate with both investors present can move outside the range bounded by $r^{(1)}$ and $r^{(2)}$. 
In order to understand this behavior of interest rates, recall that the interest rates in equilibrium should make investors indifferent between consuming now or later. Lower the investors' expected marginal utilities are the next instance [relative to its current value], the higher the equilibrium interest rate should be. In other words, the equilibrium interest rate is negatively related to the expected growth of investors' marginal utility as shown in (12). Consider an investor in the economy with utility function $e^{-p t} u\left(c_{t}\right)$ and optimal consumption process $c_{t}$. From (12), we have

$$
r_{t}=-\frac{E_{t}\left[d e^{-\rho t} u^{\prime}\left(c_{t}\right)\right]}{e^{-\rho t} u^{\prime}\left(c_{t}\right) d t}=\rho-\frac{c_{t} u^{\prime \prime}\left(c_{t}\right)}{u^{\prime}\left(c_{t}\right)} \mu_{c, t}-\frac{1}{2} \frac{c_{t}^{2} u^{\prime \prime \prime}\left(c_{t}\right)}{u^{\prime}\left(c_{t}\right)} \sigma_{c, t}^{2}
$$

where $\mu_{c, t} \equiv \frac{E_{t}\left[d c_{t}\right]}{c_{t} d t}$ and $\sigma_{c, t}^{2} \equiv \frac{E_{t}\left[d c_{t}^{2}\right]}{c_{t}^{2} d t}$ are, respectively, the expected value and the variance of the investor's consumption growth. Thus the interest rate is related to both the expected value and the variance of instantaneous consumption growth in equilibrium. High expected consumption growth implies low expected marginal utility in the future. The equilibrium interest rate then must be high. In other words, $r_{t}$ increases with the expected consumption growth. The proportionality coefficient $-\frac{c_{t} u^{\prime \prime}\left(c_{c}\right)}{u^{\prime}\left(c_{t}\right)}$ is the inverse of the elasticity of intertemporal substitution, which is also the relative risk-aversion coefficient $a\left(c_{t}\right)$ given the time-separable preferences. High variance in consumption growth, on the other hand, implies high expected marginal utility in the future by Jensen's inequality, assuming that $u^{\prime \prime \prime}\left(c_{t}\right)>0$, i.e., the marginal utility function is convex. The equilibrium interest rate then must be low. In other words, $r_{t}$ decreases with the variance of consumption growth and the proportionality constant is $\frac{c_{t} u^{\prime \prime \prime}\left(c_{t}\right)}{u^{\prime}\left(c_{t}\right)}$. Since $\frac{c_{t} u^{\prime \prime \prime}\left(c_{t}\right)}{u^{\prime}\left(c_{t}\right)}=$ $a\left(c_{t}\right)\left[1+a\left(c_{t}\right)\right]-c_{t} a^{\prime}\left(c_{t}\right)$, we can re-write the above expression as

$$
r_{t}=\rho+a\left(c_{t}\right) \mu_{c, t}-\frac{1}{2}\left\{a\left(c_{t}\right)\left[1+a\left(c_{t}\right)\right]-c_{t} a^{\prime}\left(c_{t}\right)\right\} \sigma_{c, t}^{2} .
$$

$a\left(c_{t}\right)$ in general depends on the consumption level. But for power utility functions, it is constant and $a^{\prime}\left(c_{t}\right)=0$.

When the economy is only populated by an investor with constant relative risk-aversion $a$, his consumption will be the aggregate consumption, hence $\mu_{c, t}=\mu$ and $\sigma_{c, t}^{2}=\sigma^{2}$. The interest rate will be $r(a)=\rho+a\left(\mu-\frac{1+a}{2} \sigma^{2}\right)$. For $a=1$ and $1 / 2$, we have $r^{(1)}$ and $r^{(2)}$, respectively. It is important to note that $r(a)$ is not monotonic in $a$. As $a$ increases, the elasticity of intertemporal substitution decreases which tends to increase the equilibrium interest rate. On the other hand, the risk-aversion increases which tends to decrease the equilibrium interest rate. For $a_{1}=1>a_{2}=1 / 2, r^{(1)} \geq r^{(2)}$ when $\mu \geq \frac{5}{4} \sigma^{2}$ and $r^{(1)}<r^{(2)}$ when $\mu<\frac{5}{4} \sigma^{2}$. 
When the economy is populated with both investors, each investor's consumption and marginal utility are endogenously determined. For example, when $\mu<\frac{5}{4} \sigma^{2}, r^{(1)}<r(2)$. Suppose the current level of aggregate consumption is close to 0 , the interest rate then is close to $r^{(1)}$. As $Y_{t}$ increases, investor 1 shifts his portfolio towards the risk-free security. His expected consumption growth decreases and so does its variance. The decrease in expected consumption growth tends to decrease the interest rate while the decrease in the variance of consumption growth tends to increase the interest rate. If the effect of expected consumption growth dominates, the interest rate will then be less than $r^{(1)}$ which is outside the range $\left[r^{(1)}, r^{(2)}\right]$.

We can further analyze this situation by considering the representative agent. The consumption of the representative agent is simply the aggregate endowment which is exogenously specified. His relative risk-aversion coefficient $a\left(Y_{t}\right)$ is given in (18) [with $\lambda=2 / 3$ ], which is also the inverse of his elasticity of intertemporal substitution. $a\left(Y_{t}\right)$ now varies with the consumption level. In particular, $a\left(Y_{t}\right)$ monotonically decreases with $Y_{t}, a(0)=a_{1}=1, a(\infty)=a_{2}=1 / 2$ and $a_{1}<a\left(Y_{t}\right)<a_{2}$. The interest rate given by

$$
r_{t}=\rho+a\left(Y_{t}\right) \mu-\frac{1}{2} a\left(Y_{t}\right)\left[1+a\left(Y_{t}\right)-\frac{Y_{t} a^{\prime}\left(Y_{t}\right)}{a\left(Y_{t}\right)}\right] \sigma^{2}
$$

is, however, non-monotonic in $Y_{t}$. Note that when $\mu>0$ and $\sigma^{2}=0, r_{t}$ monotonically increases with $Y_{t}$ and $r^{(2)}=\rho+\frac{1}{2} \mu<r_{t}<\rho+\mu=r^{(1)}$. When $\mu=0$ and $\sigma^{2}>0, r_{t}$ monotonically decreases with $Y_{t}$ and $r^{(1)}=\rho-\sigma^{2}<r_{t}<\rho-\frac{3}{8} \sigma^{2}=r^{(2)}$. In the general case when $\mu>0$ and $\sigma^{2}>0$, it is possible to have $a\left(Y_{t}\right) \in\left(\frac{1}{2}, 1\right)$ and $r_{t}<\min \left[r^{(1)}, r^{(2)}\right]$. Thus in our model, as $Y_{t}$ changes over time, the equilibrium interest rate can move outside the range bounded by $r^{(1)}$ and $r^{(2)}$.

The difference in the behavior of the interest rate between our model and Dumas' may be due to the difference between an exchange economy and a production economy. This is best seen by considering the two limiting cases under certainty when $\sigma=0$. In a production economy, the interest rate is simply $\mu$, independent of the preferences. This is because with production, the consumption process is endogenous. Under linear production technology, the equilibrium interest rate must equal to the intertemporal rate of transformation [as given by the production technology] which is $\mu$. In the exchange economy, the consumption path is exogenously specified. Given the consumption process, the equilibrium interest rate is $\rho+a_{i} \mu$, which does depend on investors' preferences. With positive growth [i.e., $\mu>0$ ], the interest rate increases with $a_{i}$ in this case. When there is uncertainty, the interest rate also depends (negatively) on the risk in 
future consumption. If $\sigma^{2}$ is large the interest rate decreases with $a_{i}$ due to the effect of risk aversion as discussed above. Thus, as $a_{i}$ changes, the effect on the interest rate may be negative in the production economy of Dumas (1989) while in the exchange economy considered here it is ambiguous.

Let us now consider the dynamics of instantaneous interest rate. In order to simplify our analysis, we define a new variable $\omega_{t} \equiv \widehat{c}_{1, t} / Y_{t}$, which represents investor 1 's share of aggregate consumption in equilibrium. Lemma 1 , with $\lambda=2 / 3$, implies that there exists the following one-to-one mapping between $\omega_{t}$ and $Y_{t}$ :

$$
\omega_{t}=\frac{2\left(\sqrt{1+Y_{t}}-1\right)}{Y_{t}} \text { or } Y_{t}=\frac{4\left(1-\omega_{t}\right)}{\omega_{t}^{2}}
$$

It maps $\omega_{t} \in(0,1)$ onto $Y_{t} \in(0, \infty)$ and $Y_{t}$ is monotonically decreasing with $\omega_{t} . \omega_{t} \rightarrow 1$ as $Y_{t} \rightarrow 0$ and $\omega_{t} \rightarrow 0$ as $Y_{t} \rightarrow \infty$. We can then use $\omega_{t}$ as the state variable of the economy instead of $Y_{t}$. Expressed in $\omega_{t}$, the equilibrium interest rate is

$$
r_{t}=\rho+\frac{\mu}{2-\omega_{t}}-\sigma^{2} \frac{3-2 \omega_{t}}{\left(2-\omega_{t}\right)^{3}}
$$

Hence, $r_{t}$ depends on the growth rate of aggregate consumption as well as the consumption distribution across investors. Clearly, $r_{t}$ approaches $r^{(2)}$ as $\omega_{t} \rightarrow 0$ while it approaches $r^{(1)}$ as $\omega_{t} \rightarrow 1$. Under certain parameter constraints, the interest rate is bounded below by a positive constant. For example, for $\rho>\sigma^{2}-\frac{1}{2} \mu$, we have $r_{t} \geq \rho+\frac{1}{2} \mu-\sigma^{2}>0$. Figure 1 plots $r_{t}$ as a function of $\omega_{t}$ for a specific set of parameter values. Note that $r_{t}$ reaches an interior minimum which is smaller than both $r^{(1)}$ and $r^{(2)}$ as discussed earlier.

Given the process of $Y_{t}$, the dynamics of $\omega_{t}$ can be easily obtained by applying Itô's lemma to $(22)$ :

$$
d \omega_{t}=\mu_{\omega}\left(\omega_{t}\right) d t-\sigma_{\omega}\left(\omega_{t}\right) d w_{t}
$$

where

$$
\mu_{\omega}(\omega)=-\frac{\omega(1-\omega)}{2-\omega}\left\{\mu-\sigma^{2}\left[1-\frac{\omega}{(2-\omega)^{2}}\right]\right\}, \quad \sigma_{\omega}(\omega)=\sigma \frac{\omega(1-\omega)}{2-\omega} .
$$

Similarly, we obtain the dynamics of $r_{t}$ from (23):

$$
d r_{t}=\mu_{r}\left(\omega_{t}\right) d t+\sigma_{r}\left(\omega_{t}\right) d w
$$


where

$$
\begin{aligned}
& \mu_{r}(\omega)=\left[-\frac{\mu}{(2-\omega)^{2}}+\frac{\sigma^{2}(5-4 \omega)}{(2-\omega)^{4}}\right] \mu_{\omega}+\left[\frac{\mu}{(2-\omega)^{3}}+\frac{6 \sigma^{2}(1-\omega)}{(2-\omega)^{5}}\right] \sigma_{\omega}^{2}, \\
& \sigma_{r}(\omega)=\left[-\frac{\mu}{(2-\omega)^{2}}+\frac{\sigma^{2}(5-4 \omega)}{(2-\omega)^{4}}\right] \sigma_{\omega} .
\end{aligned}
$$

Note that $\sigma_{r}(0)=\sigma_{r}(1)=0$. Thus, in the two limiting cases where there is only one investor present, the interest rate is constant and its volatility is zero. When the two investors coexist, however, the interest rate volatility is non-zero unless $r_{t}$ is at its local minimum value. Thus, preference heterogeneity among investors can increase interest rate variability. Figure 2 plots the instantaneous drift and volatility of interest rate $\sigma_{r, t}^{2}$ as a function of $\omega_{t}$. Note that in the case when there is a local minimum of $r_{t}$ as a function of $\omega_{t}$ for $\omega_{t} \in[0,1]$, the interest rate volatility drops to 0 at its local minimum as it should when it follows a diffusion process.

\subsection{Long Yield}

Let us now consider the long yield $y_{t}(\infty)$. From Theorem 2, we have the following lemma:

Lemma 5 Given the aggregate endowment process (1) and the equilibrium bond prices (19), $y_{t}(\infty)$ is a constant independent of the current value of $Y_{t}$.

As discussed earlier, when $Y_{t}$ becomes large (small), the investor with lower (higher) risk aversion dominates the economy in relative wealth and consumption. It is easy to show that for $\mu>\sigma^{2} / 2$, $Y_{t+\tau}$ will be greater than any given positive constant with probability 1 as $\tau \rightarrow \infty$, i.e., $Y_{t+\tau} \rightarrow \infty$ as $\tau \rightarrow \infty$. Thus investor 2 will eventually own the whole economy. This seems to imply that long-term bond yields should be determined mainly by the preferences of investor 2 . In other words, we should have $y(\infty)=y^{(2)}(\infty)$ which is the long-term yield when the economy is only populated with investor 2. [Here we have dropped the subscript $t$ given that the long yields are constant.] This, however, is not true as shown by the following theorem:

Theorem 4 When both investors are present in the economy, i.e., $\lambda \in(0,1)$, we have

$$
y(\infty)=\min \left[y^{(1)}(\infty), y^{(2)}(\infty)\right]
$$

where $y^{(1)}(\infty)=r^{(1)}$ and $y^{(2)}(\infty)=r^{(2)}$ are given in lemma 4 . 
When $\mu>\frac{5}{4} \sigma^{2}, y^{(1)}(\infty)>y^{(2)}(\infty)$. Then, $y(\infty)=y^{(2)}(\infty)$. When $\mu<\frac{5}{4} \sigma^{2}, y^{(1)}(\infty)<y^{(2)}(\infty)$ and $y(\infty)=y^{(1)}(\infty)$. It is important to note that the critical value is $\frac{5}{4} \sigma^{2}$ not $\sigma^{2} / 2$. Thus under mild long-run growth (i.e., $\mu<5 \sigma^{2} / 4$ ), the current long yields are still determined by the preferences of investor 1 even though his relative wealth will be negligible in the future.

This seemingly counter-intuitive result arises for the following reason. Even though in expectation investor 2 may eventually dominate the economy [in terms of his wealth and consumption], there are still possible future states of the economy in which investor 1 actually dominates. The probability of those states may be small, but the marginal utilities for consumption in these states can be high. Thus, these states can be very important in determining today's asset prices despite their small probability of occurrence. Note that for a long-term bond, it pays one unit of consumption at the maturity independent of the state of the economy then. Thus it provides an instrument to hedge against future down turns of the economy. Of course, the probability of a severe down turn in the future [leading to low consumption levels] decreases with the expected long-run growth of the economy. Under mild growth, the probability of such a down turn is non-trivial. At low levels of consumption, the marginal utility of investor 1 is much higher than that of investor 2 . Thus, a long-term bond as a hedging instrument is more attractive to investor 1 than to investor 2 . Consequently, investor 1 exerts a stronger influence on its equilibrium price. The longer the bond's maturity, the higher the expected wealth of investor 2 [relative to that of investor 1] at the maturity date and the less attractive it is to investor 2 . Thus its price will more disproportionally reflect investor 1 's preferences. Further notice that $y(\infty)$ is independent of the wealth distribution today. This implies that investors with only a small proportion of the total market wealth can have a large effect in determining asset prices.

\subsection{The Yield Curve}

For arbitrary maturity between 0 and $\infty$, the bond yield can be calculated from theorem 2 . In Figure 3, we plot the bond yields for a wide range of maturities. The parameters are set at the same values as in Figures 1 and 2: $\rho=0.02, \mu=0.05$ and $\sigma=0.20$. It then follows that $y^{(1)}(\tau)=r^{(1)}=0.0285$ and $y^{(2)}(\tau)=r^{(2)}=0.0294$. (Here, we have dropped the subscript $t$ for the two limiting yield curves since they are constant over time.] In this case, $y^{(2)}(\tau)>y^{(1)}(\tau)$ and $y(\infty)=\min \left[y^{(1)}(\infty), y^{(1)}(\infty)\right]=y^{(1)}(\infty)=0.285$ 
It is seen that at any time $t \in(0, \infty)$, the yield curve with both investors present can be downward sloping, upward sloping, or non-monotonic, depending on the current level of aggregate endowment $Y_{t}$. The yield curve has $\min \left[y^{(1)}(\infty), y^{(2)}(\infty)\right]$ as its asymptotic limit as the maturity increases. As $Y_{t}$ changes over time, the shape of the yield curve also changes. For $Y_{t}=50$ (i.e., $\omega_{t}=0.25$ ), the yield curve is downward sloping. It lies inside the range bounded by the two limiting yield curves, $\left[y^{(1)}(\tau), y^{(2)}(\tau)\right]$. The yields at short maturities are close to $y^{(2)}(\tau)$ while the yields at long maturities decrease and approach $y^{(1)}(\infty)$. For $Y_{t}=1$ (i.e., $\omega_{t}=0.83$ ), the yield curve is upward sloping. For the range of maturities shown in the figure, it lies outside the range $\left[y^{(1)}(\tau), y^{(2)}(\tau)\right]$ and is lower than $y^{(1)}(\tau)=0.28$. As maturity increases, the yield increases and approaches $y^{(1)}(\infty)$. For $Y_{t}=0.05$ (i.e., $\omega_{t}=0.99$ ), the yield curve is non-monotonic, first decreasing and then increasing as maturity increases, and lies outside the range bounded by $y^{(1)}$ and $y^{(2)}$.

Similar behavior of the yield curve is found for other parameter values. For certain parameter values and aggregate consumption level, the yield curve can exhibit a humped shape, upward sloping at short maturities and downward sloping at long maturities.

\section{Extensions and Discussions}

In previous sections, we have presented a parsimonious model of the term structure of interest rates with heterogeneous investors. For simplicity in exposition, only the case of two investors [with respectively the logarithm and square-root utility function and the same time discount parameter] and the simple endowment process is considered. In this section, we consider some extensions of the basic model.

\subsection{More General Preference Heterogeneity}

In the basic model, there are only two investors, one with logarithmic utility function and the other with square-root utility function. The equilibrium is tractable because closed-form solutions can be obtained for the optimal sharing rules given the specific preferences of the investors. Within the class of isoelastic utility functions, there are other combinations of the two risk-aversion coefficients, $a_{1}$ and $a_{2}$, that also allow closed-form solutions to the optimal sharing rule. For example, when $a_{1}>a_{2}>0$ and $a_{1}=n a_{2}$ where $n=2,3,4$, the situation is similar to the basic model and a closed-form solution can be obtained for the optimal sharing 
rule and the equilibrium.

Another extension of the model is to consider more than two investors. Again, we want to consider the situation when closed-form solutions can be obtained for the optimal sharing rule. The following three-investor economy provides such an example. All investors have isoelastic utility functions with the following exponents: $a_{1}=2, a_{2}=1$ and $a_{3}=1 / 2$. More generally, for $a_{1}>a_{2}>a_{3}>0$, closed-form solutions to the optimal sharing rule can be obtained if $\frac{a_{1}}{a_{2}}$ and $\frac{a_{1}}{a_{3}}$ belongs to the set $\{2,3,4\}$. For the case of four investors when $a_{1}>a_{2}>a_{3}>a_{4}>0$ and $\frac{a_{1}}{a_{2}}$, $\frac{a_{1}}{a_{3}}, \frac{a_{1}}{a_{4}}$ belongs to the set $\{2,3,4\}$, we can also obtain closed-form solutions. In the more general case with more than four investors within the class of power utility functions, it is more difficult to find closed form solutions to the optimal sharing rule under general wealth distributions.

Thus, we summarize the above discussion in the following theorem:

Theorem 5 Suppose that the economy consists of $I$ investors with power utility functions of the form (4). Let $a_{i}$ be the relative risk aversion of investor $i, i=1, \cdots, I$, and $0<a_{I} \leq \cdots \leq$ $a_{2} \leq a_{1}$. The optimal sharing rule has a closed-form solution if

$$
\left\{\frac{a_{1}}{a_{1}}, \frac{a_{1}}{a_{2}}, \cdots, \frac{a_{1}}{a_{I}}\right\} \subseteq\{1,2,3,4\}
$$

Furthermore, the utility function of the representative agent defined by

$$
u_{\lambda}(Y) \equiv \sup _{\sum_{i=1}^{I} c_{i}=Y} \sum_{i=1}^{I} \lambda_{i} \frac{c^{1-a_{i}}-1}{1-a_{i}}, \text { where } \lambda_{i} \geq 0 \text { and } \sum_{i=1}^{I} \lambda_{i}=1
$$

exhibits relative risk aversion that is bounded by $a_{I}$ and $a_{1}$, i.e., $a_{I} \leq-\frac{Y u_{\lambda}^{\prime \prime}(Y)}{u_{\lambda}^{\prime}(Y)} \leq a_{1}$, where $Y \in(0, \infty) .{ }^{13}$

We can easily extend our discussions on the behavior of bond yields in the two-investor case to the multiple-investor case here. The qualitative results are similar.

\subsection{More General Endowment Processes}

In the basic model, the special case of geometric Brownian motion is considered for the aggregate endowment in the two-investor economy. The simple process was chosen in order to illustrate the effect of heterogeneity in investor preferences on asset prices, in particular, bond prices and the

\footnotetext{
${ }^{13}$ The last part of the theorem was first suggested to me by Chi-fu Huang. Bruce Grundy later brought to my attention the work of Benninga and Mayshar (1993) of which this is a special case.
} 
term structure of interest rates. Since the aggregate endowment $Y(t)$ is the single variable that drives the economy, all asset prices have one explanatory factor. Price changes of bonds with different maturities are perfectly correlated. In this section, we provide some generalizations of the previous endowment process in order to relax its restrictive nature. The resulting term structures will depend on multiple factors.

For simplicity in notation, define $y_{t} \equiv \log Y_{t}$. We consider the following endowment process:

$$
\begin{aligned}
& d y_{t}=\left[\alpha-\beta y_{t}+z_{t}\right] d t+\sigma d w_{t}, \quad y_{t=0}=y_{0} \\
& d z_{t}=-z_{t} d t+\sigma_{z} d w_{z, t}, \quad z_{t=0}=z_{0}, \quad t \in[0, \infty)
\end{aligned}
$$

$w_{t}$ and $w_{z, t}$ are two independent standard Wiener process, $\alpha, \beta, \sigma$ and $\sigma_{z}$ are non-negative constants. ${ }^{14}$ The linear system $\left[y_{t}, z_{t}\right]$ contains several interesting special cases. For example, when $\beta=0$ and $z_{t} \equiv 0$ (i.e., when $z_{0}=0$ and $\sigma_{z}=0$ ), we recover the simple case considered in previous sections where $y_{t}$ follows a simple Brownian motion with constant drift $\alpha\left(\alpha=\mu-\sigma^{2} / 2\right)$. When $\beta>0$ and $z_{t} \equiv 0, y_{t}$ follows an Ornstein-Uhlenbeck process which is stationary. When $\beta=0, y_{t}$ has a drift linear in $z_{t}$. Since $z_{t}$ is stationary and has unconditional mean of zero, $\alpha$ gives the long-run growth of $y_{t}$ and $z_{t}$ the transitory growth of $y_{t}$. The aggregate consumption process in this case is quite similar to the case considered in CIR and Sun (1992) with the difference that here negative growth is allowed. ${ }^{15}$

Lemma 6 The solution to the linear system (27-28) is

$$
\begin{aligned}
y_{t+\tau} & =y_{t}+\mu_{t}(\tau)+\sigma \int_{t}^{t+\tau} e^{-\beta(t+\tau-s)} d w_{s}+\frac{\sigma_{z}}{1-\beta} \int_{t}^{t+\tau}\left[e^{-\beta(t+\tau-s)}-e^{-(t+\tau-s)}\right] d w_{z, s} \\
z_{t+\tau} & =e^{-\tau} z_{t}+\sigma_{z} \int_{t}^{t+\tau} e^{-(t+\tau-s)} d w_{z, s}
\end{aligned}
$$

where

$$
\mu_{t}(\tau) \equiv\left(\frac{\alpha}{\beta}-y_{t}\right)\left(1-e^{-\beta \tau}\right)+\frac{1}{1-\beta}\left(e^{-\beta \tau}-e^{-\tau}\right)
$$

Let $g_{t}(\tau)=Y_{t+\tau} / Y_{t}=e^{y_{t+\tau}-y_{t}}$ and

$$
v(\tau) \equiv \frac{\sigma^{2}}{2 \beta}\left(1-e^{-2 \beta \tau}\right)+\frac{\sigma_{z}^{2}}{(1-\beta)^{2}}\left\{\frac{1-e^{-2 \beta \tau}}{2 \beta}+\frac{1-e^{-2 \tau}}{2}-\frac{2\left[1-e^{-(1+\beta) \tau}\right]}{1+\beta}\right\} .
$$

\footnotetext{
${ }^{14}$ Here, for simplicity in exposition we have assumed that $w_{t}$ and $w_{z, t}$ are independent. This assumption can be easily relaxed. $z_{t}$ is assumed to be a standard Ornstein-Uhlenbeck process. There is no loss of generality by making the negative coefficient of the linear drift to be -1 .

${ }^{15}$ In the CIR model, the aggregate consumption follows a square-root process instead of the linear process assumed here which does not allow negative expected growth.
} 
Conditional on $\left[y_{t}, z_{t}\right], \log g_{t}(\tau)=y_{t+\tau}-y_{t}$ is normally distributed and $\mathrm{E}_{\mathrm{t}}\left[\log g_{t}(\tau)\right]=\mu_{t}(\tau)$, $\operatorname{Var}_{t}\left[\log g_{t}(\tau)\right]=v(\tau)$.

Let us now consider the equilibrium and bond prices under the current endowment process. Given that the state of the economy is characterized by $\left[y_{t}, z_{t}\right]$, we need more traded securities in addition to the stock and the [locally] risk-free security in order to make the financial market dynamically complete. Without further specification, we will assume that enough securities are traded so that the market is complete. Following the same steps as in section 3 , we obtain the same sharing rules between the two investors and the same pricing equations in terms of $Y_{t}$ and $g_{t}(\tau)$. We can then calculate the bond prices by applying the pricing equation (15). The results are summarized in the following lemma and theorem.

We first consider the two limiting cases. When only one of the two investors is present in the economy, the resulting term structures of interest rates are reminiscent of those in the CIR model.

Lemma 7 Given the aggregate endowment process as specified in lemma 6, the bond prices and yields in the two limiting cases are:

$$
B_{t}^{(i)}(\tau)=e^{-\rho \tau-a_{i} \mu_{t}(\tau)+\frac{1}{2} a_{i}^{2} v(\tau)}, \quad y_{t}^{(i)}(\tau)=\rho+a_{i} \mu_{t}(\tau)-\frac{a_{i}^{2}}{2} v(\tau)
$$

where $\mu_{t}(\tau)$ and $v(\tau)$ are given in lemma 6 . The corresponding the instantaneous interest rates and the long yields are

$$
r_{t}^{(i)}=\rho+a_{i}\left[\alpha-\beta y(t)+z_{t}\right]-\frac{a_{i}^{2}}{2} \sigma^{2}, \quad y^{(i)}(\infty)= \begin{cases}\rho, & \beta>0 \\ \rho+a_{i} \alpha-\frac{a_{i}^{2}}{2}\left(\sigma^{2}+\sigma_{z}^{2}\right), & \beta \leq 0 .\end{cases}
$$

where $i=1,2$.

When $\beta=0$, we obtain the special case that is very similar to the CIR model except that the interest rate follows the linear Ornstein-Uhlenbeck process here while in CIR it follows the square-root process. In this case, the growth rate of the economy depends only on $z(t)$, not on the size of the economy $y(t)$. We obtain the single factor structure for bond prices when investor preferences are homogeneous. Similar to the CIR model, the term structure can exhibit rich patterns even under this one factor structure.

Let us now consider the instantaneous interest rates, bond prices and long yields when both investors are present in the economy. 
Theorem 6 Let $\xi_{n, t}(\tau) \equiv\left\{n-1+\left[\mu_{t}(\tau)+y_{t}\right] / v(\tau)\right\} \sqrt{v(\tau)}$. Given the endowment process (2728), the bond prices with both investors present are given as follows:

$$
B_{t}(\tau)=e^{-\rho \tau}\left(\sqrt{1+Y_{t}}-1\right)\left[I_{1, t}(\tau)+I_{2, t}(\tau)\right]
$$

where

$$
\begin{aligned}
& I_{1, t}(\tau)=e^{-\xi_{1 / 2, t}(\tau) \sqrt{v(\tau)}} \\
& I_{2, t}(\tau)=e^{-\frac{\left[\mu_{t}(\tau)+\left.y_{u}\right|^{2}\right.}{2 v(\tau)}} \sum_{n=0}^{\infty} \alpha_{n}\left\{e^{\frac{1}{2} \xi_{n, t}^{2}(\tau)} \Phi\left[-\xi_{n, t}(\tau)\right]+e^{\frac{1}{2} \xi_{1 / 2-n, t}^{2}(\tau)} \Phi\left[\xi_{1 / 2-n, t}(\tau)\right]\right\}
\end{aligned}
$$

and $\alpha_{n}, n=0,1, \cdots, \Phi(\cdot)$ are defined in theorem 2. Furthermore, the instantaneous interest rate is given by

$$
r_{t}=\rho+\frac{\left(\alpha-\beta y_{t}+z_{t}\right] Y_{t}}{2 \sqrt{1+Y_{t}}\left(\sqrt{1+Y_{t}}-1\right)}-\frac{\sigma^{2} Y_{t}\left[Y_{t}^{2}-2\left(\sqrt{1+Y_{t}}-1\right)^{2}\right]}{8\left(1+Y_{t}\right)^{\frac{3}{2}}\left(\sqrt{1+Y_{t}}-1\right)^{3}}
$$

The long yield $y_{t}(\infty)$ is a constant independent of $y_{t}$ and $z_{t}$, and is given by

$$
y(\infty)=\min \left[y^{(1)}(\infty), y^{(2)}(\infty)\right]
$$

where $y^{(2)}(\infty)$ and $y^{(2)}(\infty)$ are given in lemma 7 .

The endowment process defined by (27-28) is Gaussian. It leads to normal distributions for the growth rate of the economy over any finite periods. This allows us to calculate bond prices. However, it is easy to see from our calculations that we need not restrict ourselves to only Gaussian processes. We can consider more general processes for the aggregate endowment. One example is the following square-root process:

$$
d y_{t}=\left[\alpha-\beta y_{t}\right] d t+\sigma \sqrt{y_{t}} d w_{t}, \quad y_{0} \geq 0, \quad t \in[0, \infty)
$$

where $\alpha(\geq 0)$ and $\beta$ are constants. This process is reminiscent of the process of aggregate endowment assumed in the CIR model. It is easy to show that under (29) we have

$$
r_{t}^{(i)}=\rho+a_{i} \alpha-a_{i}\left[\beta+a_{i}^{2}\right] \sigma^{2} y_{t}
$$

Since $y_{t} \geq 0, r_{t}^{(i)} \geq 0$ if $k \equiv-\beta-a_{i}^{2}>0$. Define $\widehat{r}_{t}^{(i)}=r_{t}^{(i)}-\left[\rho+a_{i} \alpha\right]$, we have

$$
d \widehat{r}_{t}^{(i)}=\left[\alpha+\frac{|\beta|}{k} \widehat{r}_{t}^{(i)}\right] d t+(\sigma / \sqrt{k}) \sqrt{\widehat{r}_{t}^{(i)}} d w_{t}
$$

$\widehat{r}_{t}^{(i)}$ will be non-negative [if it starts from non-negative values]. Cox, Ingersoll and Ross (1985a) provide a thorough examination on the properties of process (29) or (30). 
When both investors are present, $m_{t}=\frac{e^{-p t}}{\sqrt{1+e^{y_{t}}}-1}$. We can simply apply theorem 1 to derive the equilibrium interest rate. Since the probability density of $y_{t+\tau}$ conditional on $y_{t}$ (with $\tau \geq 0$ ) can be calculated in closed-form, the bond prices can be calculated in the same way as in theorem 2. We omit these calculations here.

\subsection{Stationarity}

One unattractive feature of the current model is its long-run behavior. With positive growth, the economy will eventually be dominated by the less risk-averse investor. The steady state distribution of bond yields will then simply be the one when only the less risk averse investor is present. In other words, the importance of investor heterogeneity will eventually disappear. Although stationary distributions of bond yields can be obtained in which the effect of heterogeneity remains important, it requires the stationarity of the endowment process. This feature is particularly undesirable for the empirical implementation of the model given the positive growth observed in the data.

One way to allow positive growth of the aggregate endowment and to maintain the importance of investor heterogeneity in the steady state [in terms of the distribution of bond yields] at the same time is to relax the assumption that the time discount parameters of the two investors are the same and to modify the aggregate endowment process. Let $\rho_{i}, i=1,2$, be the time discount parameter of investor $i$. It is easy to show that the optimal sharing rule now is

$$
\widehat{c}_{1, t}(Y, t)=\frac{2}{b(t)}\left[\sqrt{1+b(t) Y_{t}}-1\right], \quad \widehat{c}_{2, t}\left(Y_{t}, \lambda\right)=Y_{t}-\widehat{c}_{1, t}\left(Y_{t}, \lambda\right)
$$

where $b(t)=b_{0} e^{-2\left(\rho_{2}-\rho_{1}\right) t}$ and $b_{0}=4(1-\lambda)^{2} / \lambda^{2}$. The marginal utility of the representative agent $m_{t}$ now has the form

$$
m_{t}=\frac{e^{-\left(2 \rho_{2}-\rho_{1}\right) t}}{\sqrt{1+b_{0} e^{-2\left(\rho_{2}-\rho_{1}\right) t} Y_{t}}-1}
$$

In order to maintain the importance of investor heterogeneity in the steady state, we need $e^{-2\left(\rho_{2}-\rho_{1}\right) t} Y_{t}$ to be stationary. Instead of assuming a geometric Brownian motion for $Y_{t}$, we can assume that $Y_{t}$ is trend-stationary, i.e., $Y_{t} \equiv e^{\left(\mu-\frac{1}{2} \sigma^{2}\right) t+y_{t}}$ and $y_{t}$ follows a stationary process such as an Ornstein-Uhlenbeck process as discussed in Section 5.2. Furthermore, we assume that $\mu-\sigma^{2} / 2=2\left(\rho_{2}-\rho_{1}\right)$. Thus, for $\rho_{2}-\rho_{1}>0$ positive growth can be allowed in the model and the steady state of the equilibrium does not degenerate to the case with only one investor. ${ }^{16}$

\footnotetext{
${ }^{18}$ I thank George Constantinides for pointing out a problem with the stationary distributions of consumption
} 
It should be pointed out that this is a knife-edge case. When $\rho_{2}-\rho_{1}>0$, only the growth at $\mu=\sigma^{2} / 2+2\left(\rho_{2}-\rho_{1}\right)$ gives us the desirable behavior in the long-run. One remedy to this situation is to consider preferences that are time non-separable. For example, one can endogenize the time discount parameter by making the time discount parameter depend on past consumption. [High levels past consumption leads to large values of the time discount parameter.] The development of a detailed model of this type will not be further pursued here. For models with time nonadditive preferences under certainty, see, e.g., Koopmans (1962), Uzawa (1965), Lucas and Stokey (1984).

\section{Further Comments}

In section 4, we have only considered the prices of pure discount bonds. From (15), we can price any security given its payoff stream. In particular, we have derived the equilibrium stock price in theorem 1. It is given by the following expectation:

$$
S_{t}=S\left(Y_{t}\right)=\left[\sqrt{1+Y_{t}}-1\right]\left[\frac{1}{\rho}+\int_{0}^{\infty} e^{-\rho \tau} \mathrm{E}_{t} \sqrt{1+Y_{t} g_{t}(\tau)} d \tau\right]
$$

Clearly, the current stock price is only a function of the current level of aggregate consumption [given the initial conditions of the economy]. In the two limiting cases, the corresponding stock prices are, respectively, $S_{t}^{(1)}=S^{(1)}\left(Y_{t}\right)=Y_{t} / \rho$ and $S_{t}^{(2)}=S^{(2)}\left(Y_{t}\right)=Y_{t} /\left(r^{(2)}-\mu+\frac{1}{2} \sigma^{2}\right)$. It is easy to show that $S(Y) \rightarrow S^{(1)}(Y)$ when $Y \rightarrow 0$ and $S(Y) \rightarrow S^{(2)}(Y)$ when $Y \rightarrow \infty$. Note that from (16), $S(Y)$ satisfies the following ordinary differential equation:

$$
\frac{1}{2} \sigma^{2} S^{\prime \prime}(Y)+[\mu Y-\sigma \pi(Y)] S^{\prime}(Y)-r(Y) S(Y)+Y=0 .
$$

Thus the stock price is given by the solution to this equation with the above boundary conditions [see Wang (1994) for a more detailed discussion].

We can further price other securities in the equilibrium. For example, for a European call option on a pure discount bond, its current price $c(B, t ; K, \tau)$ is simply $\mathrm{E}_{\mathrm{t}}\left[\frac{m_{t+\tau}}{m_{t}} c(B, t+\tau ; K, 0)\right]$ where $K$ is the strike price of the option, $\tau$ the maturity of the option, $B_{t}(\tau)$ the price of the discount bond with same maturity, and $c(B, t+\tau ; K, 0)$ the terminal payoff of the option. With some algebra, the conditional expectation can be explicitly calculated.

distribution and state prices when $Y_{t}$ follows Geometric Brownian motion. 
Our basic model is presented in a continuous-time setting. This is purely for mathematical convenience. The model can also be presented in a discrete-time setting and most of the results remain the same. As pointed out by Sun (1992) in the case of the CIR model, the discretetime representation may be easier to estimate empirically, especially in the presence of general nominal shocks. Similar arguments can be made here, although a detailed discussion on this issue is outside the scope of this paper. 


\section{References}

Araujo, A., and Monteiro, P., "Equilibrium without Uniform Conditions," Journal of Economic Theory 48 (1989): 416-427.

Becker, R.A. "The Existence of Perfect Foresight Competitive Equilibrium in a Simple Dynamic Model of Capital Accumulations with Heterogeneous Households," working paper, Indiana University (1978).

Benninga, S., and Mayshar, J., "Dynamic Wealth Distribution, Trade and Asset Pricing," unpublished manuscript, Rodney L. White Center for Financial Research (1993).

Cass, D., "Optimum Growth in An Aggregate Model of Capital Accumulation," Review of Economic Studies 32 (1965): 233-240.

Constantinides, G., "Intertemporal Asset Pricing with Heterogeneous Consumers and without Demand Aggregation," Journal of Business 55 (1982): 253-267.

Cox, J., Ingersoll, J., and Ross, S., "A Theory of Term Structure of Interest Rates," Econometrica 53 (1985a): 385-408.

Cox, J., Ingersoll, J., and Ross, S., "An Intertemporal General Equilibrium Model of Asset Prices," Econometrica 53 (1985b): 363-384.

Duffie, D., Dynamic Asset Pricing Theory, New Jersey: Princeton University Press, 1992.

Duffie, D., and Huang, C.F., "Implementing Arrow-Debreu Equilibria by Continuous Trading of Few Long-Lived Securities," Econometrica 53 (1985): 1337-56.

Dumas, B., "Two-Person Dynamic Equilibrium in the Capital Market," Review of Financial Studies 2 (1989): 157-188.

Dybvig, P., and Huang, C.F., "Nonnegative Wealth, Absence of Arbitrage and Feasible Consumption Plans," Review of Financial Studies 1 (1989): 377-401.

Harrison, J.M., and Kreps, D., "Martingales and Arbitrage in Multiperiod Securities Markets," Journal of Economic Theory 20 (1979): 381-408.

Huang, C.F., “An Intertemporal General Equilibrium Asset Pricing Model: The Case of Diffusion Information," Econometrica 55 (1986):117-142.

Huang, C.F., and Pagès, H., "Optimal Consumption and Portfolio Policies with An Infinite Horizon: Existence and Convergence," unpublished manuscript, MIT Sloan School Working Paper \#3172-90-EFA (1990).

Karatzas, I., Lehoczky, J.P., and Shreve, S.E., "Existence and Uniqueness of Multi-Agent Equilibrium in A Stochastic, Dynamic Consumption/Investment Model," Mathematics of Operations Research 15 (1990): 80-128.

Karatzas, I., and Shreve, S.E., Brownian Motion and Stochastic Calculus, Springer-Verlag (1988).

Karlin, S., and Taylor, H.M., A Second Course in Stochastic Processes, Academic Press (1981).

Koopmans, T.C., "Stationary Utility and Impatience," Econometrica 28, (1962): 287-309. 
Koopmans, T.C., "On the Concept of Optimal Economic Growth," in The Econometric Approach to Development Planning, Rand McNally, Chicago, 1965.

Liptser, R.S., and Shiryayev, A.N., Statistics of Random Processes I: General Theory, SpringerVerlag (1977).

Longstaff, F., and Schwartz, E., "Interest Rate Volatility and the Term Structure: A TwoFactor General Equilibrium Model," Journal of Finance 47 (1992): 1259-82.

Lucas, R.E., Jr., "Asset Prices in an Exchange Economy," Econometrica 40 (1972): 1429-1444.

Lucas, R.E., Jr., and Stokey, N.L., "Optimal Growth with Many Consumers," Journal of Economic Theory 32 (1984): 139-171.

Mas-Colell, A., and Zame, W., "Equilibrium Theory in Infinite Dimensional Spaces," in W. Hildenbrand and H. Sonnenschein, Handbook of Mathematical Economics, Vol 4: 1835-98. Amsterdam: North-Holland, 1992.

McCulloch, J.H., "A Reexamination of Traditional Hypothesis about the Term Structure: A Comment," Journal of Finance 48 (1993): 779-790.

Merton, R.C., "Lifetime Portfolio Selection Under Uncertainty: The Continuous Time Case," Review of Economics and Statistics 51 (1969): 247-257.

Merton, R.C., "An Intertemporal Capital Asset Pricing Model," Econometrica 41 (1973): 867887.

Merton, R.C., Continuous Time Finance, Blackwell, Oxford, 1990.

Radner, R., "Existence of Equilibrium of Plans, Prices, and Price Expectations in a Sequence of Markets," Econometrica 40 (1972): 289-303.

Rubinstein, M., "An Aggregation Theorem for Security Markets," Journal of Financial Economics 3 (1974): 201-224.

Solow, R.M., "A Contribution to the Theory of Economic Growth," Quarterly Journal of Economics 70 (1956): 65-94.

Stapleton, R.C., and Subrahmanyam, M.G., "Risk Aversion and the Intertemporal Behavior of Asset Prices," Review of Financial Studies 3 (1990): 677-694.

Sun, T.S., "Real and Nominal Interest Rates: A Discrete-Time Model and Its Continuous-Time Limit," Review of Financial Studies 5 (1992): 581-612.

Uzawa, H., "Time Preference, the Consumption Function, and Optimum Asset Holdings," in Value, Capital and Growth: Papers in Honor of Sir John Hicks (J.N. Wolfe, Ed.), pp. 485-504, University of Edinburgh Press, Edinburgh, Scotland, 1968.

Vasicek, O., "An Equilibrium Characterization of the Term Structure," Journal of Financial Economics 5 (1977): 177-188.

Wang, J., "The Term Structure of Interest Rates in A Pure Exchange Economy with Heterogeneous Investors," working paper, MIT (1994). 


\section{Appendix}

In this appendix, we provide proofs to some of the results in the text. The proofs are only for the basic model. Extensions to more general endowment processes are straightforward.

For convenience, let us first give a formal definition of our economy and introduce some notation. ${ }^{17}$ The continuous-time economy is defined on the finite time span $[0, T]$. The uncertainty and the information structure are represented by a filtered [complete] probability space $(\Omega, \mathcal{F}, \mathbf{F}, P)$ on which a one-dimensional Brownian motion $w_{t}, t \in[0, T]$, is defined. The filtration $\mathbf{F}=\left\{\mathcal{F}_{t}, t \in[0, T]\right\}$ is the augmentation under $P$ of the filtration generated by $w$.

The consumption space $C_{+}$is defined as the set of positive, adapted consumption rate process that satisfy (3). The securities market consists of the [locally] risk-free security which pays a sure interest $r_{t}$ and the stock which pays dividend at rate $Y_{t}$ and is traded at [ex-dividend] price $S_{t} . Y_{t}$ is given by $(2)$. The trading strategy $(\alpha, \theta)$ is a 2-dimensional predictable process adapted to $\mathcal{F}_{t}$ where $\alpha_{t}$ denotes holdings of the risk-free security and $\theta_{t}$ holdings of the stock. A trading strategy is admissible if it satisfies condition (3) and $W_{t} \equiv \alpha_{t}+\theta_{t} S_{t} \geq 0, t \in[0, T]$. Let $\Theta$ denote the set of admissible trading strategies. A consumption/trading strategy is budget-feasible if

$$
W_{T}=W_{0}+\int_{0}^{T}\left\{\alpha_{t} r_{t} d t+\theta_{t}\left(Y_{t} d t+d S_{t}\right]-c_{t} d t\right\}
$$

Investors' preferences are given in (4) which are continuous, smooth and strictly concave. Investors' initial endowments are in shares of the stock, $\left(0, \theta_{i, 0_{-}}\right), i=1,2$ and $\sum_{i} \theta_{i, 0_{-}}=1$.

Proof of Lemma 2. Let $u_{i}(c)=E_{0}\left[\int_{0}^{T} e^{-\rho t \frac{c_{1}^{1-a_{i}}-1}{1-a_{i}} d t}\right], \partial u_{i}(c)=e^{-\rho t} c^{-a_{i}}$ and $\phi \cdot c=$ $\mathrm{E}_{0}\left[\int_{0}^{T} \phi_{t} c_{t} d t\right]$. We need to show that $\phi_{0, t}=e^{-\rho t} \frac{\sqrt{1+b Y_{0}}-1}{\sqrt{1+b Y_{t}}-1}$ is the pricing function that supports an Arrow-Debreu equilibrium given an optimum $\left(\widehat{c}_{1}, \widehat{c}_{2}\right)$. Clearly, $\phi>0$. Furthermore, $\forall c \in C_{+}$such that $u_{i}(c)>u_{i}\left(\widehat{c}_{i}\right)[i=1,2], \partial u_{i}\left(\widehat{c}_{i}\right) \cdot\left(c-\widehat{c}_{i}\right)<0$ since $u_{i}$ is strictly concave. Note that $\phi_{0, t}=\left[\sqrt{1+b Y_{0}}-1\right] \partial u_{i}\left(\hat{c}_{i, t}\right)$. Thus, $\phi \cdot c>\phi \cdot \hat{c}_{i}$. This completes the proof.

Proof of Lemma 3. In order to show that the price processes given in (12) characterize an equilibrium, we need to show that (a) $\left(\widehat{c}_{i},\left(\widehat{\alpha}_{i}, \hat{\theta}_{i}\right)\right)$ is a budget feasible consumption/trading strategy for each investor $i$ and (b) any other admissible trading strategies that yields higher

\footnotetext{
${ }^{17}$ For a reference of the terminology used here, see, e.g., Duffie (1992).
} 
expected utility is not budget feasible. For $(a)$, first note that $\left(\widehat{\alpha}_{1}, \hat{\theta}_{1}\right)$ and $\left(\alpha_{2}, \hat{\theta}_{2}\right)$ given in Theorem 1 are admissible $\left[W_{1, t}, W_{2, t}>0\right.$ and the corresponding gain processes are integrable]. Next, we need to show that $\left(\widehat{c}_{i},\left(\widehat{\alpha}_{i}, \hat{\theta}_{i}\right)\right), i=1,2$, is budget-feasible. In order to show this, we use the standard equivalent martingale approach. Let $Q$ be a measure on the space $(\Omega, \mathcal{F})$ defined by its Radon-Nikodym derivative with respect to $P: d Q / d P=\phi(T, \omega)$. Clearly, $Q$ and $P$ are equivalent measures. It can be shown that the gain process of any admissible trading strategy, defined as $\alpha r_{t} d t+\theta_{t}\left[Y_{t} d t+d S_{t}\right]$, is a martingale under $Q$. For $W_{T}=0$, the budget-feasibility of a consumption/trading policy $(c,(\alpha, \theta))$ under $Q$ becomes

$$
W_{0}=\mathrm{E}_{0}^{*}\left[\int_{0}^{T} c_{t} d t\right]
$$

where $\mathrm{E}^{*}$ denotes the expectation under $Q$. It is easy to verify that this is true for $\left(\widehat{c}_{i},\left(\widehat{\alpha}_{i}, \widehat{\theta}_{i}\right)\right)$, $i=1,2$. For (b), note that for any consumption strategy $c$ financed by $(\alpha, \theta), u_{i}(c)>u_{i}\left(\widehat{c}_{i}\right)$ implies that $\phi \cdot c>\phi \cdot \hat{c}_{i}$. Thus, $\mathrm{E}_{0}^{*}\left[\int_{0}^{T} c_{t} d t\right]>\mathrm{E}_{0}^{*}\left[\int_{0}^{T} \hat{c}_{i, t} d t\right]=W_{i, 0}$, i.e., $c$ is not budget-feasible. This completes the proof.

Proof of Theorem 2. Since $g_{t}(\tau)$ is log-normally distributed, we can write $g_{t}(\tau)=e^{\mu(\tau)+\sigma(\tau) \tilde{\epsilon}}$ where $\mu(\tau) \equiv\left(\mu-\frac{1}{2} \sigma^{2}\right) \tau, \sigma(\tau) \equiv \sigma \sqrt{\tau}$ and $\tilde{\epsilon} \sim \mathcal{N}(0,1)$. The calculation of $I_{1, t}(\tau)$ is trivial. For $I_{2, t}(\tau)$, we have

$$
\begin{aligned}
I_{2, t}(\tau)= & \frac{1}{\sqrt{2 \pi}} \int_{-\infty}^{\infty} e^{-\left[\delta_{\mathrm{t}}(\tau)+\epsilon / \sigma(\tau)\right] \sigma^{2} \tau} \sqrt{1+e^{\left[\delta_{\mathrm{t}}(\tau)+\epsilon / \sigma(\tau)\right] \sigma^{2} \tau}} e^{-\frac{1}{2} \epsilon^{2}} d \epsilon \\
= & \frac{1}{\sqrt{2 \pi}}\left\{\int_{-\infty}^{-\delta_{\mathrm{t}}(\tau) \sigma(\tau)} \sum_{n=0}^{\infty} a_{n} e^{(n-1)\left[\delta_{\mathrm{t}}(\tau)+\epsilon / \sigma(\tau)\right] \sigma^{2} \tau} e^{-\frac{1}{2} \epsilon^{2}} d \epsilon\right. \\
& \left.\quad+\int_{-\delta_{\mathrm{t}}(\tau) \sigma(\tau)}^{\infty} \sum_{n=0}^{\infty} a_{n} e^{-\left(n+\frac{1}{2}\right)\left[\delta_{\mathrm{t}}(\tau)+\epsilon / \sigma(\tau)\right] \sigma^{2} \tau} e^{-\frac{1}{2} \epsilon^{2}} d \epsilon\right\} \\
= & \sum_{n=0}^{\infty} a_{n}\left\{\frac{1}{\sqrt{2 \pi}} \int_{-\infty}^{-\delta_{\mathrm{t}}(\tau) \sigma(\tau)} e^{(n-1)\left[\delta_{\mathrm{t}}(\tau)+\epsilon / \sigma(\tau)\right] \sigma^{2} \tau} e^{-\frac{1}{2} \epsilon^{2}} d \epsilon\right. \\
& \left.\quad+\frac{1}{\sqrt{2 \pi}} \int_{-\delta_{\mathrm{t}}(\tau) \sigma(\tau)}^{\infty} e^{-\left(n+\frac{1}{2}\right)\left[\delta_{\mathrm{t}}(\tau)+\epsilon / \sigma(\tau)\right] \sigma^{2} \tau} e^{-\frac{1}{2} \epsilon^{2}} d \epsilon\right\}
\end{aligned}
$$

where $\delta_{t}(\tau) \equiv\left[\left(\mu-\frac{1}{2} \sigma^{2}\right) \tau+\log Y_{t}\right] /\left(\sigma^{2} \tau\right)$ and $a_{n}, n=0, \cdots$, are the coefficients of the Taylor expansion of $\sqrt{1+x}$. Let $\xi_{n, t}(\tau) \equiv \sigma(\tau)\left[n-1+\delta_{t}(\tau)\right]$. It is easy to show that

$$
\begin{aligned}
\int_{-\infty}^{-\delta_{\mathrm{t}}(\tau) \sigma(\tau)} e^{(n-1)\left[\delta_{\mathrm{t}}(\tau)+\epsilon / \sigma(\tau)\right] \sigma^{2} \tau} e^{-\frac{1}{2} \epsilon^{2}} \frac{d \epsilon}{\sqrt{2 \pi}} & =e^{-\frac{1}{2} \delta_{\mathrm{t}}(\tau)^{2} \sigma^{2} \tau+\frac{1}{2} \xi_{n, t}(\tau)^{2} \Phi\left[-\xi_{n}(t, \tau)\right]} \\
\int_{-\delta(t, \tau) \sigma(\tau)}^{\infty} e^{-\left(n+\frac{1}{2}\right)\left[\delta_{\mathrm{t}}(\tau)+\epsilon / \sigma(\tau)\right] \sigma^{2} \tau} e^{-\frac{1}{2} \epsilon^{2}} \frac{d \epsilon}{\sqrt{2 \pi}} & =e^{-\frac{1}{2} \delta_{\mathrm{t}}(\tau)^{2} \sigma^{2} \tau+\frac{1}{2} \xi_{1 / 2-n, t}(\tau)^{2} \Phi\left[\xi_{1 / 2-n, t}(\tau)\right]}
\end{aligned}
$$


where $\Phi(z)=\frac{1}{\sqrt{2 \pi}} \int_{-\infty}^{z} e^{-\frac{1}{2} x^{2}} d x$. Thus, we obtain the results in theorem 2 .

Proof of Theorem 4. When $\tau \gg 1, \mu(\tau) \gg 1$ and $\sigma^{2}(\tau) \gg 1$. Rewrite $I_{2, t}(\tau)$ as

$$
\begin{aligned}
I_{2, t}(\tau) & =\frac{1}{\sqrt{2 \pi}} \int_{-\infty}^{\infty} e^{-\mu(\tau)-\sigma(\tau) \epsilon-\frac{1}{2} \epsilon^{2}} \sqrt{1+Y(t) e^{\mu(\tau)+\sigma(\tau) \epsilon}} d \epsilon \\
& =\frac{1}{\sqrt{2 \pi}} e^{-\mu(\tau)+\frac{1}{2} \sigma^{2}(\tau)} \int_{-\infty}^{\infty} \sqrt{e^{-\epsilon^{\prime 2}}+e^{\mu(\tau)-\frac{3}{4} \sigma^{2}(\tau)-\left[\epsilon^{\prime}-\frac{1}{2} \sigma(\tau)\right]^{2}}} d \epsilon^{\prime}
\end{aligned}
$$

where the last equation is obtained by the change of variable $\epsilon^{\prime}=\epsilon+\sigma(\tau)$. Observing that when $d \gg 1$

$$
\begin{aligned}
0 & \leq \int_{-\infty}^{\infty} \frac{\left[\sqrt{e^{-x^{2}}}+\sqrt{c e^{-(x-d)^{2}}}\right]^{2}-\left[\sqrt{e^{-x^{2}}+c e^{-(x-d)^{2}}}\right]^{2}}{\sqrt{e^{-x^{2}}}+\sqrt{c e^{-(x-d)^{2}}}+\sqrt{e^{-x^{2}}+c e^{-(x-d)^{2}}}} d x \\
& =\int_{-\infty}^{\infty} \frac{2 \sqrt{c e^{-x^{2}} e^{-(x-d)^{2}}}}{\sqrt{e^{-x^{2}}}+\sqrt{c e^{-(x-d)^{2}}}+\sqrt{e^{-x^{2}}+c e^{-(x-d)^{2}}} d x \ll 1 .}
\end{aligned}
$$

This then implies that for $d \gg 1$,

$$
\int_{-\infty}^{\infty} \sqrt{e^{-x^{2}}+c e^{-(x-d)^{2}}} d x \approx \int_{-\infty}^{\infty}\left[\sqrt{e^{-x^{2}}}+\sqrt{c e^{-(x-d)^{2}}}\right] d x
$$

Applying this approximation to the $I_{2}(t, \tau)$ when $\tau \gg 1$, we have

$$
I_{2, t}(\tau) \approx \operatorname{Max}\left[e^{-\mu(\tau)+\frac{1}{2} \sigma^{2}(\tau)}, \sqrt{Y(t)} e^{-\frac{1}{2} \mu(\tau)-\frac{3}{8} \sigma^{2}(\tau)}\right]
$$

Theorem 4 follows then immediately.

Proof of Theorem 5. The optimal sharing rule is obtained by solving the following optimization problem:

$$
\begin{array}{ll}
\sup _{s\left\{c_{i}\right\}} & \sum_{i}^{I} \lambda_{i} \frac{c^{1-a_{i}}-1}{\gamma_{i}} \\
\text { s.t. } & c_{i} \geq 0, \forall i=1, \cdots, I, \text { and } \sum_{i=1}^{I} c_{i}=Y,
\end{array}
$$

where $a_{1} \geq a_{2} \geq \cdots \geq a_{I}>0, i=2, \cdots, I$. Given the strict concavity of the objective function and the linear constraints, there is a unique solution to the maximization problem. The corresponding first order conditions are

$$
c_{i}^{-a_{i}}=\frac{\lambda_{1}}{\lambda_{i}} c_{1}^{-a_{i}}, \quad i=2, \cdots, I
$$


Then,

$$
c_{i}=\left(\frac{\lambda_{i}}{\lambda_{1}}\right)^{\frac{1}{a_{i}}} c_{1}^{\frac{a_{1}}{a_{i}}}, \quad i=2, \cdots, I .
$$

From the resource constraint, we have

$$
\sum_{i=1}^{I}\left(\frac{\lambda_{i}}{\lambda_{1}}\right)^{\frac{1}{a_{i}}} c_{1}^{\frac{a_{1}}{a_{i}}}=Y .
$$

When $\left\{\frac{a_{1}}{a_{1}}, \frac{a_{1}}{a_{2}}, \cdots, \frac{a_{1}}{a_{1}}\right\} \subseteq\{1,2,3,4\}$, the above equation is a fourth order polynomial equation and has closed form solutions. The unique solution that guarantees $0<c_{1}<Y$ gives the optimal sharing rule. 


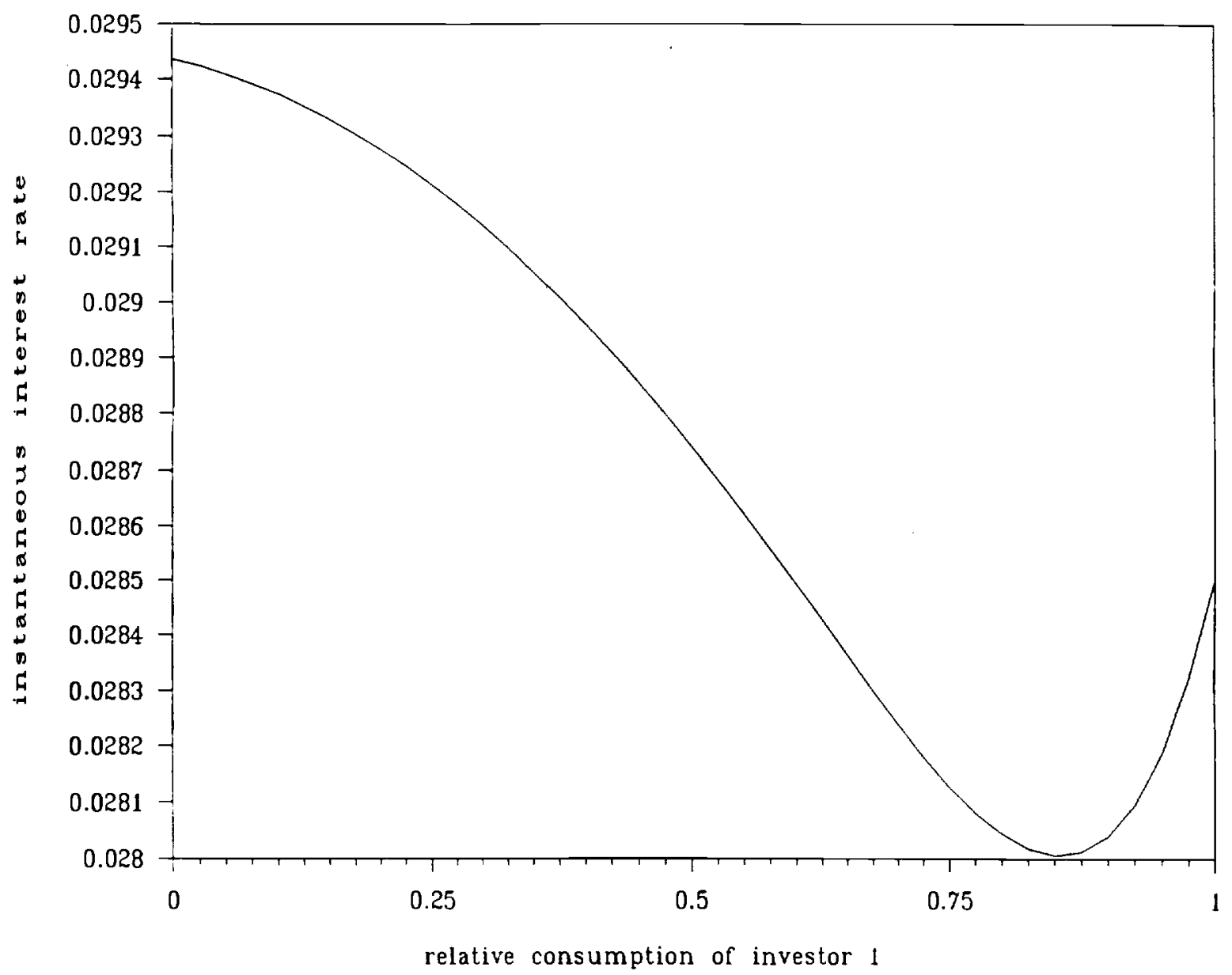

Figure 1. Instantaneous interest rate $r_{t}$ plotted as a function of $\omega_{t}$, the consumption of investor 1 relative to the aggregate consumption. The parameters are set at the following values: investors' time-discount coefficient $\rho=0.02$, expected rate of aggregate consumption growth $\mu=0.05$, instantaneous standard deviation of aggregate consumption growth $\sigma=0.20$. The instantaneous interest rate with only investor 1 present is $r^{(1)}=0.0285$ and the instantaneous interest rate with only investor 2 present is $r^{(2)}=0.0294$. 


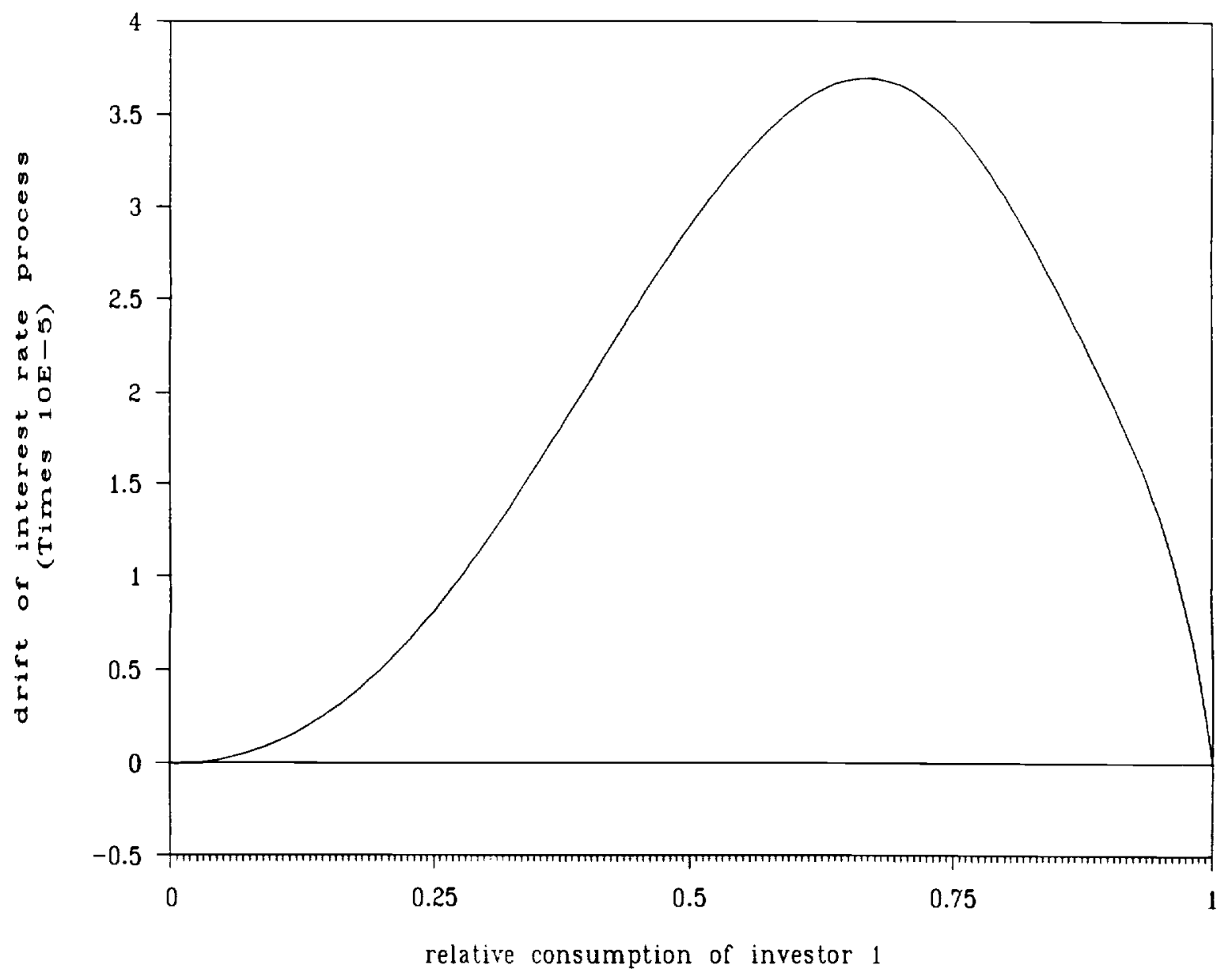

Figure 2(a). The instantaneous drift of the interest rate process $\mu_{r, t}$ plotted as a function of $\omega_{t}$, the consumption of investor 1 relative to the aggregate consumption. The parameters are set at the following values: investors' timediscount coefficient $\rho=0.02$, expected rate of aggregate consumption growth $\mu=0.05$, instantaneous standard deviation of aggregate consumption growth $\sigma=0.20$. 


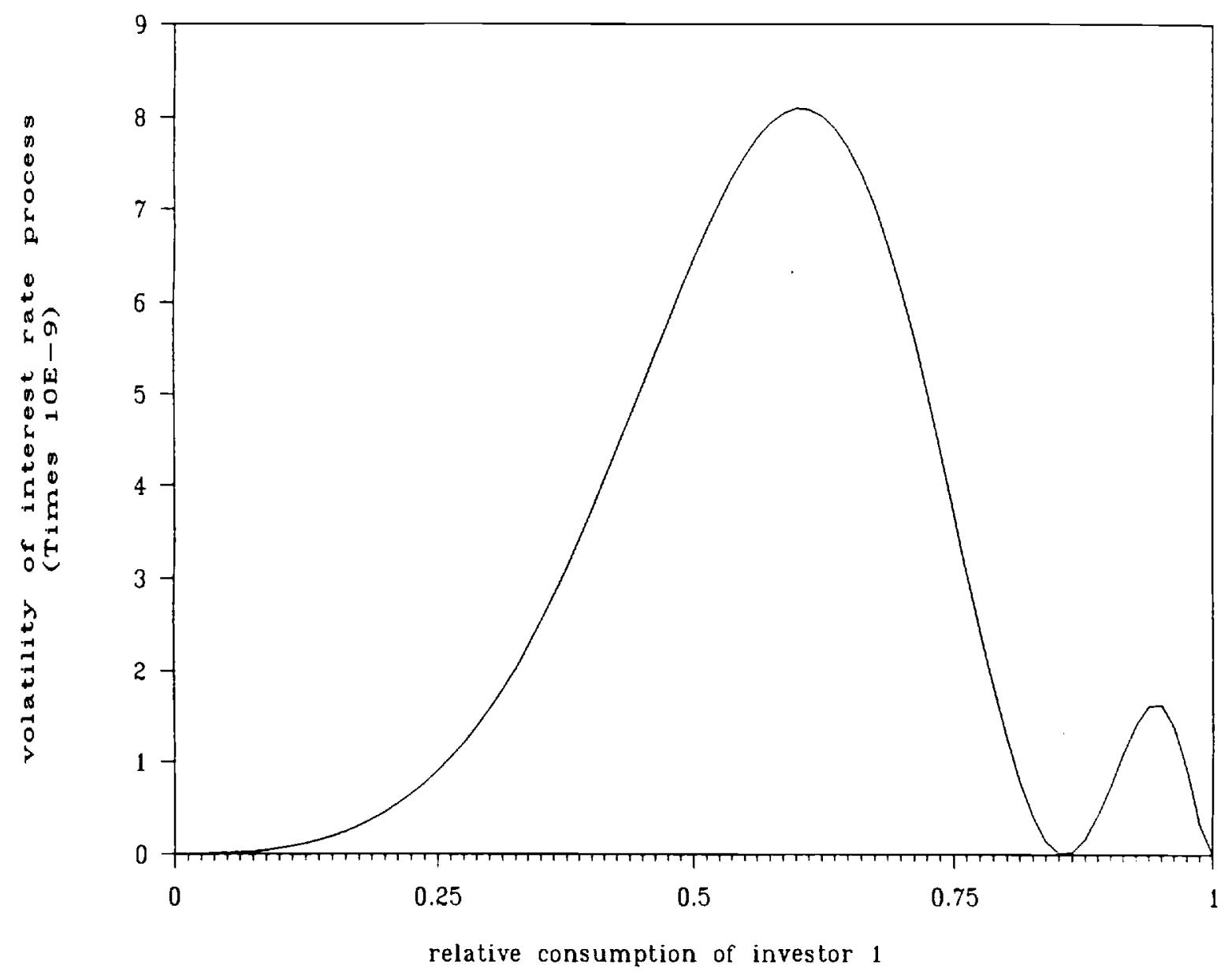

Figure 2(b). The instantaneous variance of the interest rate process $\sigma_{r, t}^{2}$ plotted as a function of $\omega_{t}$, the consumption of investor 1 relative to the aggregate consumption. The parameters are set at the following values: investors' timediscount coefficient $\rho=0.02$, expected rate of aggregate consumption growth $\mu=0.05$, instantaneous standard deviation of aggregate consumption growth $\sigma=0.20$. 


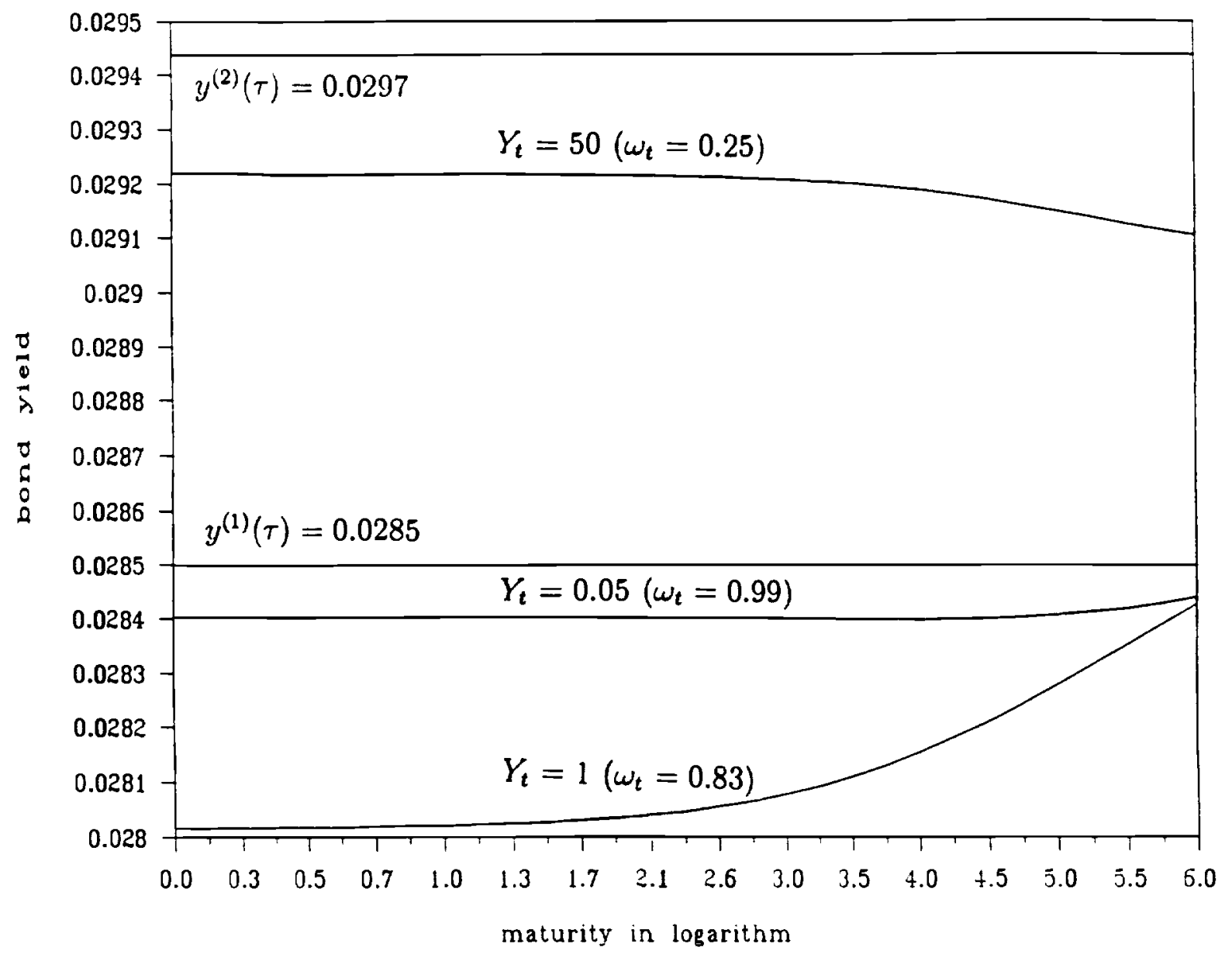

Figure 3. The bond yield $y_{t}(\tau)$ plotted against $\operatorname{logarithm}$ of maturity $\log \tau$ at different levels of current aggregate consumption $Y_{t}$ or equivalently the consumption of investor 1 relative to the aggregate consumption $\omega_{t}$. The level of $Y_{t}\left(\right.$ or $\left.\omega_{t}\right)$ is chosen to be $Y_{t}=0.05,1,50$ (or $\omega_{t}=0.99,0.83,0.25$ ), respectively. The parameters are set at the following values: investors' time-discount coefficient $\rho=0.02$, expected rate of aggregate consumption growth $\mu=0.05$, instantaneous standard deviation of aggregate consumption growth $\sigma=0.20$. The bond yield with only investor 1 present is $y^{(1)}(\tau)=0.0285$ and the bond yield with only investor 2 present is $y^{(2)}(\tau)=0.0294$, both independent of maturity. 